\title{
Metastability in Fluctuation Driven First-order Transitions: Nucleation of Lamellar Phases
}

\author{
P. C. Hohenberg \\ AT\&T Bell Laboratories \\ Murray Hill, NJ 07974 \\ J. B. Swift \\ Department of Physics and Center for Nonlinear Dynamics \\ University of Texas, Austin, TX 78712
}




\begin{abstract}
The nucleation of a lamellar phase from a supercooled homogeneous phase in a fluctuation driven first-order transition is studied, based on a phenomenological free energy due to Brazovskii. The absence of phase coexistence in the corresponding mean-field approximation makes application of the standard droplet theory of homogeneous nucleation problematic. A self-consistent coarse-graining procedure is introduced to overcome this difficulty, and the barrier height for nucleation of a critical droplet is estimated in the weak-coupling limit. Contrary to earlier estimates the critical droplet shape is shown to be anisotropic in general. Some effects of distortions and defects in the lamellar structure are considered and are shown to affect the critical droplet only very near coexistence where the probability of nucleation vanishes. The coarse-graining procedure introduced here follows from a novel application of the momentum-shell renormalization group method to systems in the Brazovskii class. Possible applications of the theory to the microphase separation transition in diblock copolymers and to Rayleigh-Bénard convection are briefly discussed.
\end{abstract}




\section{Introduction}

In 1975 Brazovskii [1] investigated isotropic or nearly isotropic systems in which the fluctuation spectrum had a minimum at a nonzero wavevector $|\mathbf{q}|=q=q_{0}$, represented by a hypersphere in $d$-dimensional reciprocal space. He found that in the symmetric case where mean-field theory would predict a continuous transition to a periodic ordered state with spatial period $2 \pi / q_{0}$, fluctuation effects lead to a discontinuous or first-order transition. In the case of weak fluctuations (small noise), the transition point is close to its mean-field value and the self-consistent Hartree approximation employed by Brazovskii could be justified as the leading term in a systematic perturbation expansion. The physical origin of the effect, which is essentially independent of system dimensionality for $d \geq 2$, lies in the large phase space for one-dimensional fluctuations in the direction transverse to the hypersphere.

Although initially proposed to describe weakly anisotropic antiferromagnets and cholesteric liquid crystals, the Brazovskii model was subsequently shown to apply to the nematic to smectic- $C$ transition in liquid crystals [2], to pion condensates in neutron stars [3], to the onset of RayleighBénard convection $[4,5]$ and most notably to the microphase separation transition in symmetric diblock copolymers [6-9]. It is in this last system that experimental confirmation of the theory was achieved [8] by quantitatively estimating the mean-field parameters and showing that the observed first-order transition was inconsistent with the predictions of mean-field theory, but was well described by the Brazovskii theory.

Given this measure of success it is natural to ask about the lifetime of the metastable isotropic phase as the system is cooled below the thermodynamic transition point [10]. From a theoretical point of view the problem is formulated by classical homogeneous nucleation theory [11] as well as by more sophisticated field-theoretic approaches [11, 12], in terms of the free energy barrier for creation of a droplet of ordered phase inside the disordered phase. The critical droplet is the one which balances the free-energy cost of the interface between coexisting phases with the gain from the bulk ordering. Mathematically, the barrier is calculated by first solving a saddle-point or mean-field equation with the boundary conditions that the system is disordered at infinity and ordered at the origin, say. Now for fluctuation driven first-order transitions quite generally [13], there is no phase coexistence in mean-field theory so this first step of the calculation cannot be taken.

Our work addresses this issue by developing a coarse-graining procedure whereby the modes with wavevectors in the range

$$
0<q<q_{0}-\Lambda, \text { and } q_{0}+\Lambda<q<\infty
$$

are averaged over, and the remaining modes with

$$
\left|q-q_{0}\right|<\Lambda
$$

are retained as fluctuating degrees of freedom. When $\Lambda \rightarrow 0$ all modes are averaged over and we recover the bulk thermodynamic averages calculated by Brazovskii [1]. For $\Lambda>0$, we obtain an effective free energy $\mathcal{F}_{\Lambda}$ for modes with wavevectors in the band (1.1b), whose average wavevector is $q_{0}$ but whose envelope can vary on a scale $L>\Lambda^{-1}$. For $\Lambda$ not too large the bulk phases described by $\mathcal{F}_{\Lambda}$ do show phase coexistence in mean-field theory, so a critical barrier height can be estimated from classical nucleation theory for given $\Lambda$. We are then left with the problem of determining the proper value of the cutoff $\Lambda$, and for this we propose a self-consistent procedure whereby $\Lambda$ is equal to the local rate of variation of the envelope in the critical droplet solution.

In cases when the ordered state is spatially anisotropic it is important to optimize the shape of the critical droplet, which will not be spherical due to the anisotropy of the interface free energy. 
The standard way to carry out this optimization is known as a Wulff construction [14] and it arises in the present problem as well. The critical droplet we are led to for a lamellar ordered state has the form of a long needle with lamellae transverse to the needle axis. In addition, just as for ordering in liquid crystals [15], we find that under some circumstances line defects can be introduced into the structure and lead to deformations of the lamellae which lower the critical barrier height. Since these effects depend on gaining surface free energy at the cost of line energy, defects are only favorable very near coexistence where the critical droplet has large dimensions and the barrier height itself is large.

In the Brazovskii theory there is a unique small parameter $\lambda$ representing the smallness of the dimensionless noise strength, or equivalently of the dimensionless coupling constant. The reduced temperature $\bar{\tau}=\bar{\tau}_{c}<0$ at which the first-order transition takes place is of order $\left|\bar{\tau}_{c}\right| \sim \lambda^{2 / 3}$ below the mean-field transition which takes place at $\bar{\tau}=0$. For a $d$-dimensional system the critical free-energy barrier for creation of an anisotropic (Wulff) droplet is found to be

$$
\begin{aligned}
& \bar{B}_{W} \sim \lambda^{(1-d) / 6}\left[\frac{\left|\bar{\tau}_{c}\right|}{|\bar{\tau}|-\left|\bar{\tau}_{c}\right|}\right]^{d-1}, \quad|\bar{\tau}| \rightarrow\left|\bar{\tau}_{c}\right|, \\
& \bar{B}_{W} \sim \lambda^{(5-d) / 2}|\bar{\tau}|^{(d-7) / 2}, \quad|\bar{\tau}| \gg\left|\bar{\tau}_{c}\right| \sim \lambda^{2 / 3} .
\end{aligned}
$$

In contrast an isotropic droplet has a barrier

$$
\begin{aligned}
& \bar{B}_{i s o} \sim \lambda^{(1-d) / 3}\left[\frac{\left|\bar{\tau}_{c}\right|}{|\bar{\tau}|-\left|\bar{\tau}_{c}\right|}\right]^{d-1}, \quad|\bar{\tau}| \rightarrow\left|\bar{\tau}_{c}\right| \\
& \bar{B}_{i s o} \sim \lambda^{(3-d)}|\bar{\tau}|^{d-4}, \quad|\bar{\tau}| \gg|\bar{\tau}|
\end{aligned}
$$

from which we see that the anisotropic barrier is always less than the isotropic one. An important conclusion of (1.2a) is that for $|\bar{\tau}| \sim\left|\bar{\tau}_{c}\right| \sim \lambda^{2 / 3}$ the dimensionless barrier $\left[\bar{B}_{W} \sim \lambda^{(1-d) / 6}\right]$ is still large for $\lambda \ll 1$ and $d \geq 2$, i.e. the probability of nucleation is low. On the other hand this is the point at which the droplet size becomes of the same order as the interface width, and for lower quenches $\left(|\bar{\tau}|>\left|\bar{\tau}_{c}\right|\right)$ the droplet will be ramified [16]. We thus do not expect spontaneous nucleation of well-defined Wulff droplets.

Although our model and methods follow those of Fredrickson and Binder [10] our conclusions are different. These authors only estimated the barrier for isotropic droplets in the domain (1.1a), and did not consider either ramified or anisotropic droplets, which according to our estimates are the ones which are likely to be nucleated.

In Sec. II the coarse-grained free energy is obtained, first by a phenomenological argument which follows the derivation of the expanded Brazovskii free energy in Ref. [10], and then by using a novel momentum-shell renormalization group. In Sec. III interface and droplet free energies are estimated, based first on the coarse-grained free energy with fixed cutoff, and then on a free energy with self-consistently determined cutoff. The self-consistency is shown not to affect the scaling of the nucleation barrier. The anisotropy of the interface free energy on the other hand is important, and it leads to an anisotropic critical droplet whose shape is determined by a Wulff construction. The contributions to the barrier height of defects and distortions of the order inside the critical droplet are estimated and it is shown that these effects are negligible except very close to coexistence, when the nucleation barrier and critical droplet dimensions are very large. Section III concludes with a brief discussion of experiment and of ways to pursue the theory in more quantitative directions. The derivations of the coarse-grained free energies are described in the Appendixes. 


\section{The Coarse-grained Brazovskii Model}

\section{A Bulk behavior}

Our starting point is a phenomenological model with relaxational (Model A) dynamics and a Brazovskii free energy which we write as

$$
\begin{aligned}
\partial_{\bar{t}} \bar{\psi}= & -\frac{\delta \overline{\mathcal{F}}}{\delta \bar{\psi}}+\bar{\eta} \\
\overline{\mathcal{F}}= & \int d^{d} \overline{\mathbf{x}}\left\{\frac{1}{2} \bar{\tau} \bar{\psi}^{2}+\frac{\lambda}{4 !} \bar{\psi}^{4}+\frac{\tilde{\xi}_{0}^{4}}{2}\left[\left(\bar{\nabla}^{2}+\bar{q}_{0}^{2}\right) \bar{\psi}\right]^{2}\right\}, \\
& \left\langle\bar{\eta}(\overline{\mathbf{x}}, \bar{t}) \bar{\eta}\left(\overline{\mathbf{x}}^{\prime}, \bar{t}^{\prime}\right)\right\rangle=2 \delta^{(d)}\left(\overline{\mathbf{x}}-\overline{\mathbf{x}}^{\prime}\right) \delta\left(\bar{t}-\bar{t}^{\prime}\right) .
\end{aligned}
$$

In the above equations the quantities $\bar{q}_{0}, \tilde{\xi}_{0}$ are considered to be of order unity and there is a single small parameter

$$
\lambda \ll 1
$$

and a control parameter $\bar{\tau}$. The static (long-time) solution has the bulk free energy

$$
\bar{\Phi}(\bar{\tau}, \lambda)=-\ln \left[\left\langle e^{-\overline{\mathcal{F}}}\right\rangle\right]
$$

where the angular brackets in (2.3) denote an average over the Gaussian noise $\bar{\eta}$, which can be represented by a functional integral [see below; the effective temperature, or noise strength, has been scaled to unity in Eq. (2.10)]. The relation between our model and various physical systems can be recovered by referring to Eq. (2) of Brazovskii [1], Eqs. (2.3) and (4.1) of Fredrickson and Binder [10], and Eqs. (A26-29) of Hohenberg and Swift [5]. The essential point is that the small coupling constant $\lambda$ reflects the smallness of the noise strength in the original systems.

According to the derivation of Brazovskii [1], for small $\lambda$ the self-consistent propagator in the disordered phase is obtained from the Hartree diagram in Fig. 1a, as

$$
\bar{g}^{-1}(\bar{q})=\bar{r}+\tilde{\xi}_{0}^{4}\left(\bar{q}^{2}-\bar{q}_{0}^{2}\right)^{2},
$$

with

$$
\bar{r}=\bar{\tau}+\frac{\lambda}{2} \int \frac{d^{d} \bar{q}}{(2 \pi)^{d}} \frac{1}{\bar{r}+\tilde{\xi}_{0}^{4}\left(\bar{q}^{2}-\bar{q}_{0}^{2}\right)^{2}} .
$$

As shown below, the solution $\bar{r}(\bar{\tau})$ of Eq. (2.5) remains positive for all $\bar{\tau}$, so the linear instability of the disordered state, signalled by the vanishing of $\bar{r}$ in mean-field theory $(\lambda=0)$, has been completely eliminated in the Hartree approximation. For $\bar{\tau}<0$ there is a competing ordered solution with [1]

$$
\langle\bar{\psi}(\overline{\mathbf{x}})\rangle=\bar{A} e^{i \overline{\mathbf{q}}_{0} \cdot \overline{\mathbf{x}}}+c c
$$

a propagator leading to

$$
\bar{r}_{A}=\bar{\tau}+\frac{\lambda}{2} \int \frac{d^{d} \bar{q}}{(2 \pi)^{d}} \frac{1}{\bar{r}_{A}+\tilde{\xi}_{0}^{4}\left(\bar{q}^{2}-\bar{q}_{0}^{2}\right)}+\lambda|\bar{A}|^{2},
$$

and a field $\bar{h}$ conjugate to $\bar{\psi}$, given by

$$
\bar{h}=\bar{A}\left(\bar{r}_{A}-\frac{1}{2} \lambda|\bar{A}|^{2}\right) .
$$


The bulk free energy difference per unit volume $\Delta \bar{\Phi}$ between the disordered and the ordered states can be obtained from the relation [1]

$$
\Delta \bar{\Phi}=\int_{0}^{\bar{A}} \frac{\partial \bar{\Phi}}{\partial \bar{A}^{\prime}} d \bar{A}^{\prime}=\int_{0}^{\bar{A}} 2 \bar{h} d \bar{A}^{\prime}=\int_{\bar{r}}^{\bar{r}_{A}} 2 \bar{h} \frac{d \bar{A}^{\prime}}{d \bar{r}^{\prime}} d \bar{r}^{\prime} .
$$

For small $\bar{r}, \bar{r}_{A}$ Eqs. (2.5) and (2.7) may be rewritten in the form

$$
\begin{aligned}
\bar{r} & =\bar{\tau}+\bar{\alpha} \lambda / \sqrt{\bar{r}}, \\
\bar{r}_{A} & =\bar{\tau}+\bar{\alpha} \lambda / \sqrt{\bar{r}_{A}}+\lambda|\bar{A}|^{2},
\end{aligned}
$$

where

$$
\bar{\alpha}=\left(\bar{q}_{0}^{d-2} S_{d} \pi\right) / 4(2 \pi)^{d} \tilde{\xi}_{0}^{2} \equiv \pi \alpha / 2,
$$

and $S_{d}$ is the surface area of the $d$-dimensional unit sphere. The essential point is that in all $d \gtrsim 2$, the integral in Eqs. (2.5) and (2.7) contributes only near the surface of the $d$-dimensional sphere of radius $\bar{q}_{0}$, and it is a one-dimensional integral in the radial direction. The transverse dimensions only contribute to the coefficient $\bar{\alpha}$. As shown by Brazovskii, the free energy difference per unit volume $\Delta \bar{\Phi}$ of Eq. (2.9) then takes the form

$$
\Delta \bar{\Phi}=-\frac{\bar{r}^{2}}{2 \lambda}-\bar{\alpha} \bar{r}^{1 / 2}+\frac{\bar{r}_{A}^{2}}{2 \lambda}+\bar{\alpha} \bar{r}_{A}^{1 / 2}+\frac{1}{4} \lambda|\bar{A}|^{4},
$$

where $\bar{r}(\bar{\tau})$ and $\bar{r}_{A}(\bar{\tau})$ are given by (2.10) and (2.11) [as mentioned in HS [5] some numerical coefficients which were incorrect in Eq. (14) of [1] have been changed]. The free energy difference changes sign at

$$
\bar{\tau}=\bar{\tau}_{c}=-2.03(\bar{\alpha} \lambda)^{2 / 3},
$$

which is the bulk (first-order) transition point in the Hartree approximation. Let us introduce the reduced variables

$$
\begin{aligned}
r & =(\alpha \lambda)^{-2 / 3} \bar{r} \\
\tau & =(\alpha \lambda)^{-2 / 3} \bar{\tau} \\
\psi & =\lambda^{1 / 2}(\alpha \lambda)^{-1 / 3} \bar{\psi}, \\
\langle\psi\rangle & =A e^{i \mathbf{q} 0 \cdot \mathbf{x}}+c c \\
A & =\lambda^{1 / 2}(\alpha \lambda)^{-1 / 3} \bar{A}, \\
\mathbf{x} & =\overline{\mathbf{x}} / \bar{\ell} \\
q_{0} & =\bar{q}_{0} \bar{\ell} \\
\bar{\ell} & =\left(2 \tilde{\xi}_{0}^{2} \bar{q}_{0}\right)(\alpha \lambda)^{-1 / 3} \equiv \bar{\xi}_{0}(\alpha \lambda)^{-1 / 3},
\end{aligned}
$$

in terms of which Eqs. (2.1b), (2.5), (2.7), (2.13) and (2.14) become

$$
\begin{aligned}
\overline{\mathcal{F}} & =\beta \mathcal{F}, \\
\beta & =\bar{\ell}^{d}(\alpha \lambda)^{4 / 3} \lambda^{-1}, \\
\mathcal{F} & =\int d^{d} x\left\{\frac{1}{2} \tau \psi^{2}+\frac{1}{4 !} \psi^{4}+\frac{1}{2}\left(4 q_{0}^{2}\right)^{-1}\left[\left(\nabla^{2}+q_{0}^{2}\right) \psi\right]^{2}\right\}, \\
r & =\tau+\pi /\left(2 r^{1 / 2}\right), \\
r_{A} & =\tau+\pi /\left(2 r_{A}^{1 / 2}\right)-\frac{1}{4}|A|^{4}, \\
\Delta \bar{\Phi} & =\frac{(\alpha \lambda)^{4 / 3}}{\lambda} \Delta \Phi=\frac{(\alpha \lambda)^{4 / 3}}{\lambda}\left[\frac{1}{2}\left(r_{A}^{2}-r^{2}\right)+\frac{\pi}{2}\left(r_{A}^{1 / 2}-r^{1 / 2}\right)-\frac{1}{4}|A|^{4}\right], \\
\tau_{c} & =-2.03(\pi / 2)^{2 / 3}=-2.74 .
\end{aligned}
$$


In the scaling of Eq. (2.15) the coupling constant in (2.18) is of order unity and the only small parameter appears in the coefficient of the gradient, $q_{0}^{-2} \sim \lambda^{2 / 3}$.

It is instructive to expand the free energy difference $\Delta \Phi$ in the order parameter $|A|^{2}$, retaining only the first three terms. The Hartree result (2.21) can be considered as a function of the independent variables $\tau$ and $|A|^{2}$ via Eqs. (2.19-20) and expanded in the form

$$
\Delta \Phi=r|A|^{2}+\frac{1}{4} u|A|^{4}+\frac{1}{36} w|A|^{6}+O\left(|A|^{8}\right) .
$$

with $r=r(\tau)$ given by the solution of Eq. 2.19), and

$$
\begin{aligned}
u & =\left(1-\pi / 4 r^{3 / 2}\right) /\left(1+\pi / 4 r^{3 / 2}\right), \\
w & =\left(9 \pi / 4 r^{5 / 2}\right) /\left(1+\pi / 4 r^{3 / 2}\right)^{3} .
\end{aligned}
$$

These functions are plotted in Fig. 2, from which it is seen that $u$ becomes negative for $\tau<0$, thus creating the first-order transition.

The approximate free energy difference (2.23) vanishes for

$$
r=r_{c}=9 u^{2} / 16 w
$$

which occurs for

$$
\tau=\tau_{c}=-2.51,
$$

which is close to the "exact" Hartree value $\tau_{c}=-2.74$ in Eq. (2.22).

Equation (2.23) is precisely the same (when allowance is made for slightly different scalings) as Eq. (2.22) of Fredrickson and Binder [10], which was arrived at by using a "Hartree potential" $\Gamma_{H}[\langle\psi\rangle]$ in place of the "bare" free-energy functional (2.18). While we believe that the proper physical interpretation of this potential is in terms of a partially coarse-grained potential $\mathcal{F}_{\Lambda}(\psi)($ as explained in the next section), for the purpose of calculating the bulk thermodynamic properties the coarse-graining can be carried out to arbitrarily long wavelengths $(\Lambda=0)$, and the potential $\Delta \Phi$ of Eq. 2.23) agrees with $\Gamma_{H}[\langle\psi\rangle]$ of Ref. [10].

Let us inquire into the domain of validity of the Hartree approximation (2.21). As mentioned by Brazovskii [1] and by Swift and Hohenberg [4], a simple estimate is obtained by finding the parameter region where the correction term in the self-energy [Fig. 1b] becomes of the same order as the terms retained in Eq. (2.5) [Fig. 1a]. Let us write Eq. (2.5) generally as

$$
\begin{aligned}
\bar{r} & =\bar{\tau}+\Sigma, \\
\Sigma & =\Sigma_{H}+\Sigma_{2}+O\left(\lambda^{3}\right),
\end{aligned}
$$

with $\Sigma_{H} \sim \lambda / \bar{r}^{1 / 2}$ the term retained in Eq. (2.5), and

$$
\Sigma_{2} \sim \lambda^{2} / \bar{r}^{3 / 2},
$$

the contribution from the diagram in Fig. 1b. In order for the perturbation expansion in $\lambda$ to be valid we require

$$
\Sigma_{2} \sim \lambda^{2} /|\bar{r}|^{3 / 2} \lesssim \bar{r}
$$

leading to $\bar{r} \gtrsim \lambda^{4 / 5}$. In this domain the Hartree $\bar{r}$, given by Eq. $(2.10)$, scales as $\bar{r} \sim(\lambda /|\bar{\tau}|)^{2}$ (i.e. $\bar{r} \ll|\bar{\tau}|$ ), and Eq. (2.31) implies

$$
|\bar{\tau}| \lesssim\left|\bar{\tau}_{G 1}\right| \sim \lambda^{3 / 5}
$$


We use the subscript $G$ in (2.32) in analogy to the Ginzburg criterion for validity of mean-field theory in critical phenomena [18]. Here, since we are dealing with a first-order transition the theory is self-consistent so long as

$$
\left|\bar{\tau}_{c}\right| \ll\left|\bar{\tau}_{G 1}\right|
$$

which holds for

$$
\lambda^{1 / 15} \ll 1 .
$$

We thus see that the strict self-consistency only holds for impractically small coupling strengths. We may also note that a less stringent criterion was invoked by Brazovskii [1], namely

$$
\Sigma_{2} \lesssim \Sigma_{1}=\Sigma_{H}
$$

which replaces Eq. (2.32) with

$$
|\bar{\tau}| \lesssim\left|\bar{\tau}_{G 2}\right| \sim \lambda^{1 / 2}
$$

and Eq. 2.34 with

$$
\lambda^{1 / 6} \ll 1
$$

\section{B Nonuniform systems}

In order to study phase competition and nucleation we must be able to describe situations where the envelope $A$ of the order parameter $\langle\psi\rangle$ of Eq. (2.15d) can vary in space, as for instance at the interface between ordered and disordered domains. For this purpose we introduce a coarse-graining procedure, whereby modes with wavevectors larger than some cutoff [19] $\Lambda$ are averaged over using the Hartree approximation of Brazovskii, and modes with wavevector less than $\Lambda$ are retained as fluctuating modes in averages such as Eq. (2.3). In particular, let us suppose that the starting free energy (2.18) involves modes with wavevectors in the range $0 \leq q \leq 2 \Lambda_{0}=2 q_{0}$, i.e.

$$
0 \leq\left|q-q_{0}\right| \leq \Lambda_{0}
$$

with $\Lambda_{0}=q_{0}$. The average in Eq. (2.3) is then a functional integral

$$
\exp [-\beta \Phi(\tau)]=\int_{\left[0, \Lambda_{0}\right]} \mathcal{D}\left[\psi_{q}\right] \exp \left\{-\beta \mathcal{F}\left[\psi_{q}\right]\right\}
$$

where the symbol below the integral indicates the inclusion of all modes $\psi(q)$ with wavevectors in the range 2.38) with upper cutoff $\Lambda_{0}$. We now define a coarse-grained free energy $\mathcal{F}_{\Lambda}$ obtained by eliminating the modes in the slice $\Lambda<\left|q-q_{0}\right|<\Lambda_{0}$. This corresponds to setting

$$
\exp [-\beta \Phi(\tau)]=\int_{[0, \Lambda]} \mathcal{D}\left[\psi_{q}\right] \exp \left\{-\beta \mathcal{F}_{\Lambda}\left[\psi_{q}\right]\right\}
$$

with

$$
\exp \left[-\beta \mathcal{F}_{\Lambda}\right] \equiv \int_{\left[\Lambda, \Lambda_{0}\right]} \mathcal{D}\left[\psi_{q}\right] \exp \left\{-\beta \mathcal{F}\left[\psi_{q}\right]\right\} .
$$

With these definitions we show below that

$$
\Phi=\mathcal{F}_{\Lambda=0},
$$

and

$$
\mathcal{F}=\mathcal{F}_{\Lambda=\Lambda_{0}}
$$


Moreover, in Appendix B we show that if the integrals implied by Eq. 2.40) are carried out using the Hartree approximation of Brazovskii and if the resulting $\mathcal{F}_{\Lambda}$ is expanded in $\psi^{2}$, then we obtain

$$
\mathcal{F}_{\Lambda}[\psi]=\int_{[0, \Lambda]} d^{d} \mathbf{x}\left\{\frac{1}{2} r(\Lambda) \psi^{2}+\frac{1}{4 !} u(\Lambda) \psi^{4}+\frac{1}{6 !} w(\Lambda) \psi^{6}+\left(\frac{1}{4 q_{0}^{2}}\right)\left[\left(\nabla^{2}+q_{0}^{2}\right) \psi\right]^{2}\right\},
$$

where the symbol below the integral again indicates that $\psi(\mathbf{x})$ has variations involving wavevectors in the range $0<\left|q-q_{0}\right|<\Lambda$. The coefficients are given by [19]

$$
\begin{aligned}
r(\Lambda, \tau) & =\tau+\phi_{1}(\Lambda, \tau), \\
u(\Lambda, \tau) & =\left[1-\phi_{2}(\Lambda, \tau)\right] /\left[1+\phi_{2}(\Lambda, \tau)\right], \\
w(\Lambda, \tau) & =12 \phi_{3}(\Lambda, \tau) /\left[1+\phi_{2}(\Lambda, \tau)\right]^{3},
\end{aligned}
$$

with $[20]$

$$
\phi_{n}(\Lambda, \tau) \equiv \int_{\Lambda}^{\infty} \frac{d k}{\left[r(\Lambda, \tau)+k^{2}\right]^{n}} .
$$

These quantities are evaluated by first solving the transcendental equation (2.45) for $r(\Lambda, \tau)$ and then inserting the result into $\phi_{2}$ and $\phi_{3}$ to obtain $u(\Lambda, \tau)$ and $w(\Lambda, \tau)$.

The derivation of Appendix B and the result in Eqs. (2.45-48) are closely related to those of Fredrickson and Binder [10], but the physical content is rather different. We are separating out the short-wavelength modes involved in generating the first-order transition and the possibility of phase coexistence $[u(\Lambda)<0]$, from the long-wavelength modes involved in building interfaces and other large distortions of the order parameter. The "Hartree potential" $\Gamma_{H}$ of Fredrickson and Binder is essentially $\mathcal{F}_{\Lambda=0}=\Phi$, which is no longer a functional of a fluctuating order parameter $\psi(q)$ where $\left|q-q_{0}\right|<\Lambda$. This potential depends only on the average order parameter $\langle\psi\rangle\left(q=q_{0}\right)$, which is only a function of $\tau$ in a uniform system. It thus seems to us that $\mathcal{F}_{\Lambda}$ rather than $\Gamma_{H}$ is the proper starting point for the evaluation of droplet free energies and metastability lifetimes, though as shown in the next section our actual results do not differ significantly from those obtained using $\Gamma_{H}$. To make a quantitative estimate of the droplet free energy by means of $\mathcal{F}_{\Lambda}[\psi]$, Eq. (2.44), we must specify the value of $\Lambda$ and this is done in Sec. IIIB.

We should also mention that in principle the coarse-graining procedure applies to the full dynamics of Eq. (2.1a), not just to the static averages such as Eq. (2.3). Since our treatment of metastability does not go beyond the estimation of "energy" barriers [21], we have not pursued this question here. Clearly, however, a more complete theory would have to take into account the effects of coarse-graining on the dynamics and on the "entropic" corrections to the barriers height coming from fluctuations about the saddle point $[11,12]$.

\section{Properties of the coarse-grained free energy}

The model (2.44-48) defines a free energy $\mathcal{F}_{\Lambda}$ which was designed to interpolate between the bare free energy $\mathcal{F}$ of Eq. (2.18) for [20] $\Lambda=\Lambda_{0}=\infty$, and the bulk average free energy $\Delta \Phi$ of Eq. (2.23) for $\Lambda=0$. Indeed, it follows from Eq. (2.48) that for $\Lambda=\Lambda_{0}=\infty, \phi_{n}(\infty) \equiv 0$, so $\mathcal{F}_{\Lambda=\infty}$ just reproduces the bare free energy $\mathcal{F}$ of Eq. (2.18). On the other hand from Eq. (2.48) for $\Lambda=0$ we have $\phi_{1}(0)=\pi / 2 r^{1 / 2}(0), \phi_{2}(0)=\pi / 4 r^{3 / 2}(0), \phi_{3}=3 \pi / 16 r^{5 / 2}(0)$, so $r(0), u(0)$ and $w(0)$ agree with the quantities $r, u, w$, respectively, defined in Eqs. (2.19), (2.24) and (2.25). Moreover, for $\Lambda=0$ there is only one mode left in the free energy in Eq. (2.44), namely the average order parameter, for which we make the Ansatz (2.15d). The free energy per unit volume then becomes precisely the expanded Hartree expression given in Eq. 2.23. 
For intermediate $\Lambda$ values we may evaluate $r(\Lambda, \tau), u(\Lambda, \tau), w(\Lambda, \tau)$ numerically from Eqs. (2.4548). The theory is of physical interest for values of $\Lambda$ which are low enough so that $r(\Lambda, \tau)>0$, implying that the disordered phase is metastable. In that case it turns out that to good accuracy we can represent the coefficients in the form

$$
\begin{aligned}
r(\Lambda, \tau) & \simeq r(0, \tau)(1+\Lambda \tau)=r(1+\Lambda \tau) \\
u(\Lambda, \tau) & \simeq u(0, \tau)=u \\
w(\Lambda, \tau) & \simeq w(0, \tau)=w
\end{aligned}
$$

results which are valid for

$$
0<\Lambda<-\tau^{-1}, \quad \tau<0
$$

\section{A momentum-shell renormalization group}

It is natural to ask whether the coarse-grained free energy obtained in the previous section could not be derived in a more standard way, using Wilson's momentum-shell renormalization group [22, 23], for example. It turns out that because the ordering in the Brazovskii model (2.1b) involves condensation onto the surface of a sphere in reciprocal space, the usual methods are difficult to implement, and various authors have found it necessary to introduce modifications of the model in order to obtain renormalization group recursion relations [24, 25]. However, recent work on the renormalization group for Fermi liquids at low temperatures, where the wavevectors of the important modes also lie on a sphere, suggests that a direct perturbation expansion might work. Making use of some of the techniques developed by Shankar [26] for the Fermi liquid we have been able to derive recursion relations for the Brazovskii model (2.1b), keeping essentially the same type of Hartree diagrams as in earlier work. The derivation is summarized in Appendix A, and the result is again of the form (2.44), where now $r(\Lambda), u(\Lambda)$ and $w(\Lambda)$ are defined by differential recursion relations which are quoted in Eqs. (A.24). Solutions of these equations yield coefficients which are close to those of the simple approximation (2.45-48) for $\tau>0$, and show a similar dependence on $\Lambda$ and $\tau$ for $\tau<0$. There is, however, an important difference, in that the solutions of the differential recursion relations (A.24) are not defined for all $\Lambda$ and $\tau$ due to singularities for $\tau<0$, where $r(\Lambda, \tau)+\Lambda^{2}=0$. In particular the quantity $r(0, \tau)$ vanishes at a finite $\tau_{1}<\tau_{c}$, unlike the solution of (2.45) which remains positive for all $\tau$. These singularities make it difficult to use the renormalization group to estimate droplet free energies for sufficiently negative $\tau$, so we shall rather use the phenomenological coefficients (2.45-48), which are defined for all $\Lambda$ and $\tau$. Nevertheless, the recursion relations are well behaved for larger $\tau$ (including part of the metastable range), and they are of intrinsic interest, so we have presented their derivation in Appendix A.

\section{Droplet Theory of Nucleation}

\section{A Phenomenological theory}

We shall be interested in estimating the free-energy barrier [21] for nucleation of a critical droplet of the ordered phase (2.15d) embedded in the disordered phase $\psi=0$ for $\tau<\tau_{c}$. Having obtained an effective free-energy $\mathcal{F}_{\Lambda}$ with $u<0$, we first estimate the barrier height for a critical droplet with fixed $\Lambda$, deferring to the next subsection the question of the proper choice of $\Lambda$. As is done in standard nucleation theory [11-13], we seek a saddle-point solution for $\mathcal{F}_{\Lambda}$, i.e. a localized solution of the differential equation

$$
\frac{1}{\left(4 q_{0}^{2}\right)}\left(\nabla^{2}+q_{0}^{2}\right)^{2} \psi+r(\Lambda) \psi+\frac{1}{3 !} u(\Lambda) \psi^{3}+\frac{1}{5 !} w(\Lambda) \psi^{5}=0
$$


with $\psi=0$ for $|\mathbf{x}| \rightarrow \infty$ and $\psi \neq 0$ in the interior. In this equation the coefficients $r, u, w$ are $O(1)$ and are given by Eqs. (2.46-49), and $q_{0} \sim \lambda^{-1 / 3} \gg 1$. Given a solution $\psi_{s}$ of the above saddle-point equation, the classical nucleation barrier [21] is

$$
\bar{B}=\bar{B}_{\Lambda}=\beta \mathcal{F}_{\Lambda}\left[\psi_{s}\right]
$$

Although we have now reduced the calculation to a straightforward problem in differential equations, which could certainly be attacked numerically, Eq. (3.1) is still quite difficult to solve accurately, and we shall rather attempt to estimate the value of $B_{\Lambda}$ analytically, in particular its dependence on the parameters, which are $q_{0}, r, u$ and $w$ [in the remainder of this subsection we consider these parameters to be basic, and suppress their dependence on $\lambda, \Lambda$ and $\tau$ ]. The difficulty in finding a general solution of Eq. (3.1) arises from the existence of three widely different characteristic lengths. The first is the wavelength $q_{0}^{-1}$ of the ordered phase, which in the present scaling (2.15) is vanishingly small. Then there is the thickness of the interface between the ordered and disordered phases, which is of order unity (see below, however), and finally the droplet size, which diverges at coexistence where the bulk free energies of the two phases are equal.

\section{Interfaces}

We begin by considering only ordered states of the form

$$
\psi(x, \mathbf{y})=A(x, \mathbf{y}) e^{i q_{0} x}+c c,
$$

describing small distortions of a pattern consisting of parallel planes (or lines in two dimensions), with wavevector in the $x$-direction, say. Then for solutions whose envelope $A(x, \mathbf{y})$ varies slowly in space the latter can be shown to satisfy the well-known amplitude equation [27]

$$
\left[\partial_{x}+\left(i / 2 q_{0}\right) \partial_{\mathbf{y}}^{2}\right]^{2} A(x, \mathbf{y})=2 r A+u|A|^{2} A+\frac{1}{6} w|A|^{4} A,
$$

where $\mathbf{y}$ here denotes the $(d-1)$-dimensional vector transverse to the direction $x$ of alignment of the structure. For bulk states, with $A=$ constant, and for $u<-(4 r w / 3)^{1 / 2}$, the equation has two types of solutions, disordered $[A=0]$ and ordered $\left[A= \pm A_{0}\right]$ with

$$
A_{0}^{2}=3\left[\left(u^{2}-4 r w / 3\right)^{1 / 2}-u\right] / w .
$$

Their free energies become equal when $r=r_{c}$, with $r_{c}$ given by (2.26), though now $r, u, w$ denote $r(\Lambda), u(\Lambda), w(\Lambda)$ rather than $r(0), u(0), w(0)$, as in that equation.

Let us now consider interfaces between the ordered and disordered states. Clearly there are two types of simple interfaces, longitudinal and transverse. A longitudinal interface is a solution of Eq. (3.4) in which $A$ only depends on $x$ with $A=0$ for $x \rightarrow-\infty$, and $A=A_{0}$ for $x \rightarrow+\infty$ say (see Fig. 3). This is a quintic Ginzburg-Landau equation as discussed, for example, by Fredrickson and Binder [10]; the interface thickness is of order

$$
\xi_{\|} \sim(2 r)^{-1 / 2}
$$

and the interface free-energy (per unit area) is of order

$$
\sigma_{\|} \sim \xi_{\|} f_{0}
$$


where $f_{0}$ is related to the bulk free energy difference (per unit volume)

$$
\begin{aligned}
\Delta \Phi & =-f_{0} \delta \\
\delta & =\left(r_{c}-r\right) / r_{c} \\
f_{0} & \simeq 81|u|^{3} / 32 w^{2} .
\end{aligned}
$$

[If $f_{0}$ is defined by Eq. (3.9) then (3.7) is strictly correct only for $\delta \rightarrow 0$, but it is quite accurate up to $\delta=1$, and we shall use Eqs. (3.7-9) for all $\delta$ in our estimates.]

A transverse interface, on the other hand (Fig. 4), is a solution of

$$
0=\frac{1}{4 q_{0}^{2}} \partial_{y}^{4} A(y)+2 r A+u|A|^{2} A+\frac{1}{6} w|A|^{4} A,
$$

which goes to $A_{0}$ for $y \rightarrow+\infty$ and to zero for $y \rightarrow-\infty$, say. The interface thickness is now of order

$$
\xi_{\perp} \sim\left[8 q_{0}^{2} r\right]^{-1 / 4} \sim \lambda^{1 / 6} r^{-1 / 4},
$$

(which is small at fixed $r$ for $\lambda \ll 1$ ), and the interface energy is

$$
\sigma_{\perp} \sim \xi_{\perp} f_{0} \ll \sigma_{\|}
$$

It is this anisotropy in the interface energies which leads to a preference for nonspherical droplets, as detailed in the next subsections (this was missed by Fredrickson and Binder [10]).

\section{Critical droplets: isotropic case}

Let us now estimate the free energy of a critical droplet solution of Eq. (3.1). Near coexistence (i.e. for $\delta \ll 1$ ), the dimensions of the droplet are very large, and we can use the standard separation [12] of $\mathcal{F}$ into bulk and surface contributions to estimate its free energy. We first consider an isotropic droplet, i.e. one made up of concentric lamellae (we refer to three-dimensional structures in our discussion, but the results are applicable in $d=2$ also). Then the edge of the droplet is a purely longitudinal interface [see Fig. 5a], and the energy of a droplet of radius $R$ can be written in the standard way as

$$
\Delta \mathcal{F}(R) \simeq R^{(d-1)} \sigma_{\|}-R^{d} f_{0} \delta
$$

The critical droplet is the one with maximum free energy (as a function of $R$ ), namely it has radius

$$
R_{i s o} \sim \xi_{\|} / \delta
$$

and corresponds to a free energy barrier

$$
B_{\text {iso }}=\xi_{\|}^{d} f_{0} / \delta^{d-1} \sim\left[r^{-d / 2}|u|^{3} / w^{2}\right] / \delta^{d-1} .
$$

As usual the radius of the critical droplet and the barrier height diverge at coexistence, $r \rightarrow r_{c}$, $\delta \rightarrow 0$. On the other hand, as the undercooling $\delta$ grows the radius decreases and when $\delta=O(1)$ the droplet size $R$ is of the same order as the interface thickness $\xi_{\|}$, and the above estimate base on a separation between bulk and surface free energies becomes questionable. As pointed out by Unger and Klein [16] in an analogous situation, the critical barrier height may still be large at that point and it is useful to extend the calculation to the "ramified" case $\delta=O(1)$.

Let us first recast the above calculation in terms of the free energy $\mathcal{F}_{\Lambda}$, Eq. (2.44) and the associated Euler-Lagrange equation (3.1). For an isotropic configuration made up of concentric 
lamellae the wavevector of the ordered state is radial, so the order parameter may be taken in the form

$$
\psi=A(\rho) e^{i q_{0} \rho}+c c
$$

with real $A$, where $\rho$ is the radial coordinate (we neglect all transverse variation for this simple estimate). The equation for the droplet takes the form

$$
-2 \partial_{\rho}^{2} A-\frac{4}{\rho} \partial_{\rho} A+2 r A+u A^{3}+\frac{w}{6} A^{5}=0,
$$

in leading order where terms neglected are of order $\left(\rho q_{0}\right)^{-1} \partial_{\rho}^{2} A, q_{0}^{-1} \partial_{\rho}^{3} A$, or higher. The corresponding free energy is to leading order (in three dimensions)

$$
\mathcal{F}_{i s o}[A]=S_{3} \int d \rho \rho^{2}\left\{\left(\partial_{\rho} A+\rho^{-1} A\right)^{2}+r A^{2}+\frac{u}{4} A^{4}+\frac{w}{36} A^{6}\right\} .
$$

Let us make a change of variables

$$
\begin{aligned}
A & =(r /|u|)^{1 / 2} \tilde{A} \\
\rho & =r^{-1 / 2} \tilde{\rho},
\end{aligned}
$$

whereby (3.17) and (3.18) become, for $u<0$

$$
\begin{aligned}
2 \partial_{\tilde{\rho}}^{2} \tilde{A} & +(4 / \tilde{\rho}) \partial_{\tilde{\rho}} \tilde{A}-2 \tilde{A}+\tilde{A}^{3}-(3 / 32)(1-\delta) \tilde{A}^{5}=0, \\
\mathcal{F}_{i s o} & =S_{d} r^{(4-d) / 2}|u|^{-1} \tilde{\mathcal{F}}_{i s o}[\tilde{A}]
\end{aligned}
$$

and for $d=3$

$$
\tilde{\mathcal{F}}_{i s o}[\tilde{A}]=\int d \tilde{\rho} \tilde{\rho}^{2}\left\{\left(\partial_{\tilde{\rho}} \tilde{A}+\tilde{\rho}^{-1} \tilde{A}\right)^{2}+\tilde{A}^{2}-\frac{1}{4} \tilde{A}^{4}+(1 / 64)(1-\delta) \tilde{A}^{6}\right\} .
$$

where Eq. (3.8) was used. [An analogous formula for $\tilde{\mathcal{F}}$ can be derived for arbitrary $d$ ].

We now seek a (critical droplet) solution of Eq. (3.20) which vanishes for $\tilde{\rho} \rightarrow \infty$ and is nonzero for $\tilde{\rho}=0$, with $\partial_{\tilde{\rho}} \tilde{A}=0$ for $\tilde{\rho}=0$. The only parameter left in Eqs. $(3.20)$ and $(3.22)$ is $\delta$, and for $\delta \ll 1$ the solution reaches the bulk value $\tilde{A}^{2}=\tilde{A}_{0}^{2}=8$ [see Eq. (3.5)] at $\tilde{\rho}=0$. The position $\tilde{R}$ of the interface results from a balance between the surface and bulk terms in $\tilde{\mathcal{F}}$, Eq. (3.22), and an argument analogous to the one in Eq. (3.13) leads to the results $\tilde{\mathcal{R}} \sim \delta^{-1}, \tilde{B}_{\text {iso }}=\tilde{\mathcal{F}}_{\text {iso }}[\tilde{A}] \sim \delta^{-2}$ for $\delta \ll 1$. From Eq. (3.21) we then see that

$$
B_{\text {iso }}=r^{(4-d) / 2}|u|^{-1} \tilde{B}_{\text {iso }} \sim r^{(4-d) / 2}|u|^{-1} \delta^{-(d-1)},
$$

which agrees with Eq. (3.15) since for $\delta \ll 1, r \approx r_{c} \sim|u|^{2} / w$.

Let us now consider the case $\delta=O(1)$ when $\tilde{R}=O(1)$ and $\tilde{A}(\tilde{\rho}=0)$ no longer reaches the bulk value $\tilde{A}_{0}(\delta)$ corresponding to (3.5). There is still expected to be a unique solution of Eq. (3.18) satisfying the boundary conditions, and since all coefficients in (3.18) are of order unity, the barrier is given by $\tilde{B}_{i s o}=\tilde{\mathcal{F}}_{i s o}[\tilde{A}]=O(1)$. This corresponds to the "ramified" droplet of Unger and Klein [16], for which the separation between bulk and surface is not applicable, but a well-defined barrier can still be calculated from Eq. (3.20). Thus for arbitrary $\delta$ we have

$$
B_{\text {iso }} \sim r^{(4-d) / 2}|u|^{-1} \tilde{B}_{\text {iso }}(\delta),
$$

with $\tilde{B}_{i s o}(\delta)$ an $O(1)$ function proportional to $\delta^{1-d}$ for $\delta \ll 1$. 


\section{Anisotropic droplets: the Wulff construction}

All of the above estimates were based on the assumption of an isotropic solution (3.14) of the saddle-point equation (3.1), with concentric lamellae (or rolls in $d=2$ ). Since, however, the cost $\sigma_{\perp}$ of a transverse interface is less than that of a longitudinal interface $\sigma_{\|}$for small $\lambda$ [see Eq. (3.12)], we may guess that an anisotropic solution of (3.1) will lead to a lower barrier. The simplest such solution has parallel lamellae with wavevector in the $x$-direction, say, as in Eqs. (3.3) and (3.4).

Let us first consider the classical nucleation regime $\delta \ll 1$ and seek a solution of Eq. (3.1) which vanishes for $x,|\mathbf{y}| \rightarrow \infty$ and reaches the bulk value (3.5) in the center [see Fig. 5b]. Because of the anisotropy of the interface energy we must optimize not only the size but also the shape of the droplet, using the well-known Wulff construction [14]. In order to do this we consider a parallellipiped of length $\gamma \sigma_{\|}$in the $x$-direction and $\gamma \sigma_{\perp}$ in the $(d-1)$ transverse $(\mathbf{y})$ directions, with $\gamma$ an unknown constant to be determined (see Fig. 6). The volume of the figure is $\Omega=\gamma^{d} \sigma_{\perp}^{d-1} \sigma_{\|}$ so

$$
\gamma=\left(\Omega / \sigma_{\perp}^{d-1} \sigma_{\|}\right)^{1 / d}
$$

Then the total free energy contribution from all the surfaces is (see Fig. 6)

$$
\begin{aligned}
\mathcal{F}_{S} & =2 \sigma_{\|}\left(\gamma \sigma_{\perp}\right)^{d-1}+2(d-1) \sigma_{\perp}\left(\gamma \sigma_{\|}\right)\left(\gamma \sigma_{\perp}\right)^{d-1}, \\
& =2 d f_{0} \Omega^{(d-1) / d}\left(\xi_{\|} \xi_{\perp}^{d-1}\right)^{1 / d},
\end{aligned}
$$

while the bulk contribution is $\mathcal{F}_{B}=-f_{0} \Omega \delta$ [in deriving Eq. (3.26), Eqs. (3.6) and 3.12) were used]. We now maximize the total energy $\mathcal{F}=\mathcal{F}_{S}+\mathcal{F}_{B}$ with respect to the unknown volume $\Omega$, and find the "Wulff" values

$$
\Omega=\Omega_{W} \sim R_{W}^{d}
$$

with

$$
R_{W} \sim \xi_{W} / \delta \sim \sigma_{W} / f_{0} \delta \sim\left(\xi_{\|} \xi_{\perp}^{d-1}\right)^{1 / d} / \delta
$$

and a total free energy barrier

$$
B_{W} \sim \xi_{\|} \xi_{\perp}^{d-1} f_{0} / \delta^{d-1} \sim \xi_{W}^{d} f_{0} / \delta^{d-1}
$$

The longitudinal and transverse dimensions are

$$
\begin{aligned}
\left(R_{\|}\right)_{W} & \sim \gamma_{W} \sigma_{\|} \sim \xi_{\|} / \delta \\
\left(R_{\perp}\right)_{W} & \sim \gamma_{W} \sigma_{\perp} \sim \xi_{\perp} / \delta
\end{aligned}
$$

i.e. there is an anisotropy

$$
\left(R_{\|}\right)_{W} /\left(R_{\perp}\right)_{W} \sim q_{0}^{1 / 2} \sim \lambda^{-1 / 6}
$$

which diverges for $\lambda \ll 1$. Note also that the droplet becomes ramified $\left(R_{\perp} \sim \xi_{\perp}\right.$ and $\left.R_{\|} \sim \xi_{\|}\right)$for $\delta \sim 1$, just as in the isotropic case (3.14).

In order to relate the above estimate to the fundamental equation (3.1), we note that for real $A$ Eq. (3.4) takes the form

$$
\partial_{x}^{2} A+\left(4 q_{0}^{2}\right)^{-1} \nabla_{\mathbf{y}}^{4} A=2 r A+u A^{3}+\frac{1}{6} w A^{5} .
$$

We seek a solution which vanishes for $|x|,|\mathbf{y}| \rightarrow \infty$, and reaches a nonzero value over a finite region surrounding the origin. Equation (3.32) is derived from a free energy

$$
\mathcal{F}_{\text {ani }}[A]=\int d x d \mathbf{y}\left\{\left(\partial_{x} A\right)^{2}+\frac{1}{4 q_{0}^{2}}\left(\nabla_{\mathbf{y}}^{2} A\right)^{2}+r A^{2}+\frac{1}{4} u A^{4}+\frac{1}{36} w A^{6}\right\} .
$$


We now introduce the scaling of Eq. (3.19a) and

$$
\begin{aligned}
x & =r^{-1 / 2} \tilde{x}, \\
\mathbf{y} & =r^{-1 / 4}\left(2 q_{0}\right)^{-1 / 2} \tilde{\mathbf{y}},
\end{aligned}
$$

which changes Eqs. (3.32) and (3.33) into

$$
\begin{aligned}
\partial_{\tilde{x}}^{2} \tilde{A} & +\partial_{\tilde{\mathbf{y}}}^{4} \tilde{A}=2 \tilde{A}-\tilde{A}^{3}+(3 / 32)(1-\delta) \tilde{A}^{5} \\
\mathcal{F}_{a n i} & =r^{(7-d) / 4}|u|^{-1}\left(2 q_{0}\right)^{(1-d) / 2} \tilde{\mathcal{F}}_{a n i}, \\
\tilde{\mathcal{F}}_{a n i} & =\int d \tilde{x} d \tilde{\mathbf{y}}\left\{\left(\partial_{\tilde{x}} \tilde{A}\right)^{2}+\left(\partial_{\mathbf{y}}^{2} \tilde{A}\right)^{2}+\tilde{A}^{2}-\frac{1}{4} \tilde{A}^{4}+(1 / 64)(1-\delta) \tilde{A}^{2}\right\} .
\end{aligned}
$$

In the classical nucleation region $\delta \ll 1$ the solution of Eq. (3.35) depends sensitively on $\delta$, and the Wulff construction leading to Eq. (3.29) tells us that in the scaling of Eq. (3.34) we have $\tilde{\mathcal{F}}_{\text {ani }} \sim \delta^{1-d}, \tilde{R}_{\|} \sim \tilde{R}_{\perp} \sim \delta^{-1}$. The solution becomes ramified $\left(\tilde{R}_{\|} \sim \tilde{R}_{\perp} \sim 1\right)$ when $\delta=O(1)$ and in that case once again $\tilde{\mathcal{F}}_{\text {ani }} \sim O(1)$. The free energy barrier $(3.29)$ may now be written as

$$
B_{W} \sim \xi_{\|} \xi_{\perp}^{d-1} f_{0} / \delta^{d-1} \sim q_{0}^{(1-d) / 2} r^{-(1+d) / 4}|u|^{3} / w^{2} \delta^{d-1} \sim q_{0}^{(1-d) / 2} r^{(7-d) / 4} /|u| \delta^{d-1}, \delta \ll 1,
$$

where we have again used the fact that for $\delta \ll 1, r \sim r_{c} \sim|u|^{2} / w$. More generally we write, as in Eq. (3.24),

$$
B_{W} \sim q_{0}^{(1-d) / 2} r^{(7-d) / 4}|u|^{-1} \tilde{B}_{W}(\delta),
$$

with $\tilde{B}_{W}(\delta)=O(1), \tilde{B}_{W} \sim \delta^{1-d}$ for $\delta \ll 1$.

\section{B Self-consistent theory}

Having estimated the barrier height for the simplest isotropic and anisotropic critical droplets using the phenomenological model (2.44) with given $q_{0}, r, u$ and $w$, we now address the issue of the proper choice of the coarse-graining scale $\Lambda$. The reason for introducing a coarse-grained free energy in the first place was that we needed to allow for variations of the order parameter on length scales of the order of the interface width $\xi$ and larger. Thus, in order to estimate the longitudinal surface free-energy $\sigma_{\|}$we need to impose the constraint

$$
\Lambda^{-1} \lesssim \xi_{\|}
$$

It is apparent from Fig. 7 that if $\mathbf{q}$ is confined to a shell of thickness $2 \Lambda$ around the point $q_{0} \hat{\mathbf{x}}$, then for $q_{0} \gg 1$ the transverse momentum $\left|\mathbf{q}_{y}\right|=q_{y}$ is limited by $\Lambda_{\perp}=\left(2 q_{0} \Lambda\right)^{1 / 2}$. Thus, since $\xi_{\perp}=\left(2 q_{0} \xi_{\|}\right)^{1 / 2}$ the constraint (3.40) automatically ensures that

$$
\Lambda_{\perp}^{-1} \lesssim \xi_{\perp}
$$

which is also a necessary condition for evaluating $\sigma_{\perp}$ consistently from $\mathcal{F}_{\Lambda}$. In order to estimate bulk contributions to the droplet free energy, on the other hand, we need to let $\Lambda \rightarrow 0$ as in Eq. (2.23). These different constraints can be implemented concurrently by using a type of local-density or Thomas-Fermi approximation, whereby $\Lambda$ is adjusted self-consistently to be equal to the local longitudinal rate of variation of the envelope $A(x, \mathbf{y})$ :

$$
\Lambda=\Lambda_{A}(x) \equiv A^{-1} \partial_{x} A .
$$


We thus seek an extremum of the free energy functional

$$
\mathcal{F}_{\text {ani }}^{s c}[A ; \Lambda]=\int d x d \mathbf{y}\left\{\left(\partial_{x} A\right)^{2}+\left(\frac{1}{4 q_{0}^{2}}\right)\left(\nabla_{\mathbf{y}}^{2} A\right)^{2}++r(\Lambda) A^{2}+\frac{1}{4} u(\Lambda) A^{4}+\frac{1}{36} w(\Lambda) A^{6}\right\},
$$

with $\Lambda$ determined self-consistently at each point via Eq. (3.42). It can be shown that the EulerLagrange equation is no longer precisely given by Eq. (3.4), but rather by

$$
\partial_{x}^{2} A-\left(4 q_{0}^{2}\right)^{-1} \nabla_{\mathbf{y}}^{4} A=2 \hat{r}(\Lambda) A+\hat{u}(\Lambda) A^{3}+\frac{1}{6} \hat{w}(\Lambda) A^{5},
$$

with

$$
\begin{aligned}
\hat{r}(\Lambda) & =r(\Lambda)+\Lambda d r / d \Lambda, \\
\hat{u}(\Lambda) & =u(\Lambda)+\Lambda d u / d \Lambda, \\
\hat{w}(\Lambda) & =w(\Lambda)+\Lambda d w / d \Lambda .
\end{aligned}
$$

Referring to Eqs. (2.49) we see that $d u / d \Lambda$ and $d w / d \Lambda$ are small, while $d r / d \Lambda \sim \tau$ so we set

$$
\begin{aligned}
\hat{r}(\Lambda) & =r(0)[1+2 \Lambda \tau] \\
\hat{u}(\Lambda) & =u(\Lambda)=u, \\
\hat{w}(\Lambda) & =w(\Lambda)=w .
\end{aligned}
$$

The self-consistent droplet is given by the solution of Eq. (3.44), subject to the constraint (3.42) or equivalently by the saddle point of (3.43), subject to the same constraint. For the isotropic droplet Eq. (3.42) is replaced by

$$
\Lambda_{A}(\rho)=A^{-1} \partial_{\rho} A,
$$

and the gradient terms in $(3.43)$ and $(3.44)$ are replaced by $\left[\partial_{\rho} A+(2 / \rho) A\right]^{2}$ and $\left[\partial_{\rho}^{2} A+(2 / \rho) \partial_{\rho} A\right]$, respectively, as in Eqs. (3.17) and (3.18).

We have not solved these equations numerically, but we may once again use dimensional arguments to estimate the effect of the self-consistency on the barrier height. In the isotropic case and for $\delta \ll 1$ we argue that the main change compared to Eq. (3.15) is to select $\Lambda=\xi_{\|}^{-1}$ in evaluating the surface term, whereas we may take $\Lambda=R^{-1} \sim \delta \sim 0$ in the bulk term. Thus the barrier is changed to

$$
B_{i s o}^{s c} \sim \xi_{\|}\left(\hat{r}\left(\xi_{\|}^{-1}\right)\right) f_{0}(\hat{r}(0)) / \delta^{d-1} .
$$

Now from Eq. (3.46a) and the relation $\xi_{\|}(\hat{r}) \sim \hat{r}^{-1 / 2}$ we may show that

$$
\xi_{\|}\left(\hat{r}\left(\xi_{\|}^{-1}\right)\right) \sim 3.2 \xi_{\|}(\hat{r}(0))=3.2 \xi_{\|},
$$

i.e. the self-consistency increases the barrier by a factor of order unity. More generally, for arbitrary $\delta$, we introduce the scaling (3.19), but where now $r$ and $u$ stand for $r(\Lambda=0), u(\Lambda=0)$. Then Eqs. (3.20) and (3.22) become, respectively

$$
2 \partial_{\tilde{\rho}}^{2} \tilde{A}+(2 / \tilde{\rho}) \partial_{r \tilde{h} o} \tilde{A}-2 \tilde{r}(\tilde{\Lambda}) \tilde{A}+\tilde{A}^{3}-(3 / 32)(1-\delta) \tilde{A}^{5}=0,
$$

and

$$
\tilde{\mathcal{F}}_{i s o}^{s c}[\tilde{A}]=\int d \tilde{\rho} \tilde{\rho}^{2}\left\{\left(\partial_{\tilde{\rho}} \tilde{A}+\tilde{\rho}^{-1} \tilde{A}\right)^{2}+\tilde{\tilde{r}}(\tilde{\Lambda}) \tilde{A}^{2}-\frac{1}{4} \tilde{A}^{4}+(1 / 64)(1-\delta) \tilde{A}^{2}\right\}
$$


with

$$
\begin{aligned}
\delta & =\left[r(\Lambda=0)-r_{c}\right] / r_{c}=\left(r-r_{c}\right) / r_{c} \\
\tilde{r}(\tilde{\Lambda}) & =1+\tilde{\Lambda} r^{1 / 2} \tau \\
\tilde{r}(\tilde{\Lambda}) & =1+2 \tilde{\Lambda} r^{1 / 2} \tau .
\end{aligned}
$$

The self-consistency relation (3.47) becomes

$$
\tilde{\Lambda}=\tilde{\Lambda}_{\tilde{A}}(\tilde{\rho})=\tilde{A}^{-1} \partial_{\tilde{\rho}} \tilde{A}
$$

Once again all coefficients in (3.50) are of order unity for $\delta=O(1)$, so Eq. (3.23) becomes

$$
B_{i s o}^{s c} \sim r^{(4-d) / 2}|u|^{-1} \tilde{B}_{i s o}^{s c}(\delta),
$$

where $r, u$ and $\delta$ refer to $\Lambda=0$, and $\tilde{B}_{i s o}^{s c}=O(1)$ is obtained by solving (3.50) subject to the constraint (3.55).

In the anisotropic case the arguments go through in the same way, and they lead to the replacement of $(3.29)$ for $\delta \ll 1$, by

$$
B_{W}^{s c} \sim \xi_{\|}\left[r\left(\Lambda=\xi_{\|}^{-1}\right)\right] \xi_{\perp}^{d-1}\left[r\left(\Lambda=\xi_{\|}^{-1}\right)\right] f_{0}\left[r\left(\Lambda=R_{\|}^{-1}\right)\right] \delta^{1-d} .
$$

Now for $\delta \ll 1, R_{\|}^{-1} \sim \delta \rightarrow 0$, and once again Eqs. (2.49) and (3.6a) lead to

$$
r\left(\Lambda=\xi_{\|}^{-1}\right)=0.1 r(\Lambda=0) .
$$

and

$$
\xi_{\|}\left(\Lambda=\xi_{\|}^{-1}\right)=3.2 \xi_{\|}(\Lambda=0) .
$$

Thus the self-consistent anisotropic barrier is increased with respect to (3.29) by a factor of order unity, and Eq. (3.38) still holds, with $r, u$ and $\delta$ now referred to $\Lambda=0$.

For $\delta$ of order unity the scaling of Eqs. (3.19a) and (3.34) goes through with $r=r(\Lambda=0)$, and Eqs. (3.35) and (3.37) are replaced by

$$
\begin{aligned}
\partial_{\tilde{x}}^{2} \tilde{A} & +\partial_{\tilde{y}}^{4} \tilde{A}=2 \tilde{r}(\tilde{\Lambda}) \tilde{A}-\tilde{A}^{3}+(3 / 32)(1-\delta) \tilde{A}^{5} \\
\tilde{\mathcal{F}}_{\text {ani }}^{s c}[\tilde{A}] & =\int d \tilde{x} d \mathbf{y}\left\{\left(\partial_{\tilde{x}} \tilde{A}\right)^{2}+\left(\partial_{\mathbf{y}}^{2} \tilde{A}\right)^{2}+\tilde{\tilde{r}}(\tilde{\Lambda}) \tilde{A}^{2}-\frac{1}{4} \tilde{A}^{4}+(1 / 64)(1-\delta) \tilde{A}^{6}\right\},
\end{aligned}
$$

with the self-consistency relation

$$
\tilde{\Lambda}=\tilde{\Lambda}_{\tilde{A}}(\tilde{x})=\tilde{A}^{-1} \partial_{\tilde{x}} \tilde{A},
$$

and $\tilde{r}(\tilde{\Lambda}), \tilde{\tilde{r}}(\tilde{\Lambda})$ given by Eqs. (3.53) and (3.54), respectively. Then the self-consistent estimate of the anisotropic barrier has the same form as Eq. (3.39),

$$
B_{W}^{s c} \sim q_{0}^{(1-d) / 2} r^{(7-d) / 4}|u|^{-1} \tilde{B}_{W}^{s c}(\delta),
$$

but now $r, \delta$ and $u$ refer to $\Lambda=0$, and $\tilde{B}_{W}^{s c}(\delta)=O(1)$ is obtained by solving the self-consistent equations (3.59-61).

The final results may then be expressed in terms of the original variables of Eq. (2.1b), namely $\bar{\tau}$ and $\lambda$, by using the formulas of Sec. IIA. We defer a detailed examination of the results until Sec IIID below, but it should already be clear that the anisotropic barrier (3.62) will always be smaller than the isotropic one (3.56), due to the factor $q_{0}^{(1-d) / 2} \sim \lambda^{(d-1) / 6} \ll 1$ and the higher power of $r \lesssim 1$ in Eq. (3.62). 


\section{Defects and distortions}

The calculation of the anisotropic barrier was based on the simplest ansatz designed to take advantage of the favorable transverse interface energy $\sigma_{\perp}$, namely parallel lamellae with wavevector $\mathbf{q} \simeq q_{0} \hat{\mathbf{x}}$, as in Eq. (3.3). It should be clear, however, that under certain circumstances a lowerenergy configuration can be achieved by distorting the lamellae in regions where they form (unfavorable) longitudinal interfaces, in order to gain additional surface free energy. In general such distortions tend to produce defects, whose free energy cost is linear in the size of the structure, so distortions are typically favored for large droplets.

Our discussion of defects and distortions draws heavily on the work of Cross [28] and of Fournier and Durand [15]. In particular, the latter authors showed that for smectic liquid crystals it is advantageous to introduce defects in the form of focal conics, and to pack these into overall spherical shapes in the asymptotic limit of an infinite droplet (Fig. 8). As shown below, the same arguments hold for the present system but it turns out that focal conic defects are only favorable in a small region near coexistence $\left(\delta \ll 1, \tau \rightarrow \tau_{c}, R \rightarrow \infty\right)$. Similarly, we have considered overall distortions of the anisotropic droplet into an annulus, in order to eliminate the costly longitudinal interfaces at the tips (see Fig. 9). This introduces dislocations in the bulk of the structure, however, and according to our estimates it is not favorable for any value of $\tau$.

Unless otherwise noted we will consider three-dimensional systems in this section, though similar arguments can be given for $d=2$, or any other $d>3$. Following Cross [28] let us write the gradient term in the free energy $(2.18)$ in the form

$$
\mathcal{F}_{G} \equiv \frac{1}{8 q_{0}^{2}} \int d^{3} \mathbf{x}\left[\left(\nabla^{2}+q_{0}^{2}\right) \psi\right]^{2}=\frac{1}{2} \int d^{3} \mathbf{x} \kappa\left[(\nabla \cdot \hat{\mathbf{n}})^{2}+4(\delta q)^{2}\right]
$$

where

$$
\begin{aligned}
\psi & =A e^{i \zeta}+c c, \\
\nabla \zeta & =\left(q_{0}+\delta q\right) \hat{\mathbf{n}}
\end{aligned}
$$

and the bending constant is given by

$$
\kappa=\frac{1}{2} A^{2}
$$

in these units.

Let us now consider the effect of inserting a focal conic into the Wulff droplet, as sketched in Fig. (8a,b), and let us denote by $L$ the average radius of curvature of the bend in the structure. This length also corresponds to the length of the core of the disclination defect line, which has an average cross section $\xi_{\|}^{2}$. Then as argued by Fournier and Durand [15], the introduction of the focal conic yields a bulk energy cost of bending of order $\kappa L^{-2}$ per unit volume (or $\kappa L$ overall), and a term $\kappa L \ln \left(L / \xi_{\|}\right)$to account for the core of the defect. The surface energy, on the other hand, is decreased by an amount $\sim \Delta \sigma L^{2}$ where

$$
\Delta \sigma=\sigma_{F C}-\sigma_{W}
$$

is the difference between the structures in Figs. $8 \mathrm{~b}$ and $5 \mathrm{~b}$, respectively. Now according to the calculation in Sec. IIIA3 we have $\sigma_{W} \sim f_{0}\left(\xi_{\|} \xi_{\perp}^{2}\right)^{1 / 3}$, whereas for the structure with a focal conic most of the interface is transverse, so $\sigma_{F C} \sim f_{0} \xi_{\perp}^{3} \ll \sigma_{W}$. The free energy difference between the two structures scales as

$$
\mathcal{F}_{W}-\mathcal{F}_{F C} \sim \kappa L\left[c_{1}+c_{2} \ln \left(L / \xi_{\|}\right)\right]+\Delta \sigma L^{2}
$$


where $c_{1}$ and $c_{2}$ are constants of order unity. The above quantity vanishes for a size $L$ of order

$$
L_{0}=\kappa /|\Delta \sigma| \sim \kappa / \sigma_{W},
$$

which means that it is favorable to introduce focal conics into the Wulff droplets for $R_{W}=$ $\sigma_{W} / f_{0} \delta>L_{0}$, i.e. for

$$
\delta<\delta_{F C} \sim \frac{\sigma_{W}}{f_{0} L_{0}} \sim \frac{\sigma_{W}^{2}}{f_{0} \kappa} \sim q_{0}^{-2 / 3}
$$

where we have used the relations $\sigma_{W} \sim \xi_{W} \sim\left(\xi_{\|}\right) \xi_{\perp}^{1 / 3}$, Eqs. (3.66) and (3.11) and the fact that in the present scaling and near coexistence $(\delta \ll 1)$ all the coefficients $r \sim r_{c}, u, w, f_{0}, \kappa \sim A^{2} \sim r / u$ are of order unity and only factors of $\delta$ and $q_{0} \sim \lambda^{-1 / 3} \gg 1$ need to be considered. In the range (3.70) the barrier is then determined by $\sigma_{\perp}$, i.e. it scales as

$$
B_{F C} \sim \xi_{\perp}^{3} / \delta^{2} \sim q_{0}^{-3 / 2} / \delta^{2}, \quad \delta<q_{0}^{-2 / 3}
$$

rather than (3.38).

The above calculation assumed that the Wulff droplet was unchanged in shape and size, and only the texture of the lamellae was modified. Following Ref. [15], we may consider a composite droplet consisting of a spherical array of conical domains, each one of which contains a focal conic defect (see Fig. 8d). Such a shape was found to predominate for large droplets in the case of smectic liquid crystals [15]. The surface free energy is now still transverse and it is achieved over a spherical surface $\left(\sigma_{\perp} R^{2}\right)$, since the cost is entirely in line energy $(\sim R \ln R)$ and is negligible for large $R$. Thus the free energy is given by

$$
\mathcal{F}_{s p h} \sim \sigma_{\perp} R^{2}-\delta f_{0} R^{3}
$$

leading to

$$
R_{s p h} \sim \xi_{\perp} / \delta .
$$

This is more favorable than the single conic of Fig. (8a,b) for $R_{s p h}>L_{0}$, i.e.

$$
\delta<\delta_{s p h}=\sigma_{\perp} / f_{0} L_{0} \sim \sigma_{\perp} \sigma_{W} \sim q_{0}^{-5 / 6} .
$$

The barrier for this droplet has the same scaling as the one with a single focal conic, but presumably with a smaller coefficient in the range (3.74),

$$
B_{s p h} \sim q_{0}^{-3 / 2} \delta^{-2}, \quad B_{s p h}<B_{F C}, \quad \delta<q_{0}^{-5 / 6} .
$$

Finally, let us estimate the free energy barrier for creation of an annular droplet (Fig. 9), which lowers the surface energy at the cost of splay and/or defect energy in the bulk. We first consider an undefected structure in which the bending of the lamellae leads to a change in the local wavevector, and a bulk energy cost given by the last term in Eq. (3.63). If we denote the radius by $R_{1}$ and the width by $R_{2} \ll R_{1}$, then the change in wavevector from the inner to the outer rim is $\delta q \sim q_{0} R_{2} / R_{1}$, and the energy cost is $(\delta q)^{2} R_{2}^{2} R_{1} \sim q_{0}^{2} R_{2}^{4} / R_{1}$. The surface energy is entirely transverse, so the total energy of the annulus is

$$
\mathcal{F}_{\text {annu }} \sim q_{0}^{2} R_{2}^{4} / R_{1}+q_{0}^{-1 / 2} R_{1} R_{2}-R_{1} R_{2}^{2} \delta,
$$

where near coexistence $\left(r \sim r_{c}, \delta \ll 1\right)$ we may again set $f_{0} \sim 1, \sigma_{\perp} \sim \xi_{\perp} \sim q_{0}^{-1 / 2}$. In order to find the critical size and shape we need to maximize (3.76) at fixed ratio

$$
\eta=R_{2} / R_{1}
$$


and then minimize the result with respect to the ratio $\eta$. We find $\eta_{c} \sim \delta^{1 / 2} q_{0}^{-1}, R_{1 c} \sim q_{0}^{1 / 2} \delta^{-3 / 2}$ and a barrier

$$
B_{\text {aпnu }} \sim q_{0}^{-1 / 2} \delta^{-5 / 2},
$$

which is always larger than the Wulff value $B_{W} \sim \sigma_{W} / \delta^{2} \sim \xi_{\perp}^{2} / \delta^{2} \sim 1 / q_{0} \delta^{2}$, for $\delta \lesssim 1$.

Thus the cost of changing the local wavevector is too high so we shall keep the constraint $q \approx q_{0}$ on average, and attempt to achieve bend by introducing dislocations in the bulk of the structure (Fig. 9b). Since dislocations comes form lines in three dimensions or points in two dimensions, the $2 \mathrm{~d}$ estimate of Cross [28] for the number $N_{D}$ of dislocations applies in $3 \mathrm{~d}$ also, namely

$$
N_{D} \sim q_{0} R_{2}
$$

Each dislocation costs an energy $f_{0} \delta \xi_{\|} \xi_{\perp}$ per unit length of core and has length $R_{2}$, so the bulk energy cost of the dislocations is $\sim f_{0} \delta \xi_{\|} \xi_{\perp} R_{2}^{2} q_{0}$. Thus the structure in Fig. 9b has energy

$$
\mathcal{F}_{D} \sim \delta q_{0}^{1 / 2} R_{2}^{2}+q_{0}^{-1 / 2} R_{1} R_{2}-R_{1} R_{2}^{2} \delta
$$

The same procedure as above then yields $\eta_{c} \sim\left(q_{0} \delta\right)^{-1}, R_{1 c} \sim q_{0}^{1 / 2}$, and a barrier

$$
B_{D} \sim\left(q_{0}^{1 / 2} \delta\right)^{-1}
$$

The above estimates are only valid if $R_{2}<R_{1}$, i.e. $\eta_{c}<1$ which means

$$
\delta>q_{0}^{-1} \text {. }
$$

In the next section we put together all the estimates to find the most favorable structure for each region of $\delta$ (or $\tau$ ).

\section{Results}

In Eqs. (3.56), (3.62), (3.75), 3.78) and (3.81) we have presented estimates for the barrier heights of the droplets shown in Figs. (5a,b), (8b,d) and (9b), expressed in the scaled units of Eq. (2.15), as a function of $q_{0} \sim \lambda^{-1 / 3}$ and the bulk parameters $r, u, w, \delta=\left(r_{c}-r\right) / r_{c}$ (evaluated at $\Lambda=0$ ), which depend on $\tau$. Near the bulk transition $(\delta \ll 1)$ only the parameters $\delta \ll 1$ and $q_{0} \sim \lambda^{-1 / 3} \gg 1$ survive since $r \sim r_{c}, u$ and $w$ are $O(1)$, and

$$
\delta=\left(r_{c}-r\right) / r_{c} \approx\left(|\tau|-\left|\tau_{c}\right|\right) /\left|\tau_{c}\right|=\left(|\bar{\tau}|-\bar{\tau}_{c} \mid\right) /\left|\bar{\tau}_{c}\right| \ll 1, \quad|\bar{\tau}| \rightarrow\left|\bar{\tau}_{c}\right| .
$$

Far below the transition $\left(r \rightarrow 0, \delta \rightarrow 1,|\tau| \gg 1,|\bar{\tau}| \gg\left|\bar{\tau}_{c}\right|\right)$ we have

$$
r(\tau) \sim|\tau|^{-2} \sim\left(\left|\bar{\tau}_{c}\right| /|\bar{\tau}|\right)^{2} \sim \lambda^{4 / 3}|\bar{\tau}|^{-2}, \quad|\bar{\tau}| \gg\left|\bar{\tau}_{c}\right| .
$$

Let us first compare the Wulff droplet with the various defected structures discussed in the previous section. According to Eqs. (3.70) and (3.74) the focal conic and spherical structures are favored over the Wulff droplet for $\delta<q_{0}^{-2 / 3} \sim \lambda^{2 / 9}$, and $\delta<q_{0}^{-5 / 6} \sim \lambda^{5 / 18}$, respectively. Comparing the barriers $B_{F C} \sim B_{s p h}$ in Eqs. (3.71) and (3.75) with the barrier for the defected annulus $B_{D}$ in (3.81), we see that the annulus would be favorable for $\delta<q_{0}^{-1} \sim \lambda^{1 / 3}$, but this is precisely the region given by Eq. (3.82) where the annulus is no longer well defined. We conclude that it is only in a vanishingly small region near coexistence (when the droplet size diverges and the barrier heights are very large) that defects come into play, and when they do it is in the form of focal conic structures (see Fig. 10). 
We are thus left with the Wulff droplet as the preferred one over most of the metastable range of $\tau$. In order to estimate the probability of nucleation we need the barrier $\bar{B}_{W}$ in the original units of Eq. (2.1b), since the lowest order estimate for this probability is the saddle-point contribution $\exp \left[-\bar{B}_{W}\right]$ (the noise in Eq. (2.10) is normalized to unity). According to Eqs. 2.15h), (2.16), (2.17) and (3.39) we have

$$
\bar{B}_{W}=\beta B_{W} \sim \lambda^{(1-d) / 3} q_{0}^{(1-d) / 2} r^{(7-d) / 4}|u|^{-1} \tilde{B}_{W}(\delta) .
$$

Noting that $\tilde{B}_{W}(\delta) \sim \delta^{1-d}$ for $\delta \ll 1\left(r \rightarrow r_{c}\right)$, and $\tilde{B}_{W}(\delta)=O(1)$ for $\delta=O(1)$, and taking into account the asymptotic estimates in Eq. (3.83) we find

$$
\begin{aligned}
\bar{B}_{W} & \sim \lambda^{(1-d) / 6}\left[\frac{\left|\bar{\tau}_{c}\right|}{|\bar{\tau}|-\left|\bar{\tau}_{c}\right|}\right]^{d-1}\left|\bar{\tau}^{-1} \rightarrow\right| \bar{\tau}_{c} \mid, \\
\bar{B}_{W} & \sim \lambda^{(1-d) / 6}, \quad|\bar{\tau}| /\left|\bar{\tau}_{c}\right|=O(1), \\
\bar{B}_{W} & \sim \lambda^{(5-d) / 2}|\bar{\tau}|^{(d-7) / 2}, \quad|\bar{\tau}| \gg\left|\bar{\tau}_{c}\right| \sim \lambda^{2 / 3} .
\end{aligned}
$$

A similar calculation for the isotropic droplet of Eq. (3.56) yields

$$
\begin{aligned}
\bar{B}_{i s o} & \sim \lambda^{(1-d) / 3}\left[\frac{\left|\bar{\tau}_{c}\right|}{|\bar{\tau}|-\left|\bar{\tau}_{c}\right|}\right]^{d-1}, \quad|\bar{\tau}| \rightarrow\left|\bar{\tau}_{c}\right|, \\
\bar{B}_{i s o} & \sim \lambda^{(1-d) / 3}, \quad|\bar{\tau}| /\left|\bar{\tau}_{c}\right|=O(1), \\
\bar{B}_{i s o} & \sim \lambda^{(3-d)}|\bar{\tau}|^{d-4}, \quad|\bar{\tau}| \gg\left|\bar{\tau}_{c}\right| .
\end{aligned}
$$

From the above estimates it is clear that the anisotropic Wulff droplet has a lower barrier than the isotropic droplet, but the barrier nevertheless is still large $\left[\lambda^{(1-d) / 6} \gg 1\right]$ up to and beyond the region $|\bar{\tau}| \approx\left|\bar{\tau}_{c}\right|$ when the droplet becomes ramified according to Eq. (3.30). Indeed, we may estimate the barrier height at $|\bar{\tau}| \sim\left|\bar{\tau}_{G 1}\right| \sim \lambda^{3 / 5}$, where according to Eq. (2.32) the Hartree approximation breaks down, and we find

$$
\bar{B}_{W}\left(\bar{\tau}_{G 1}\right) \sim \lambda^{(2-d) / 5} .
$$

Thus for $d=3$ the Wulff barrier is still large $\left(\lambda^{-1 / 5} \gg 1\right)$ at this point, which in reduced units corresponds to the region $\left(|\tau| \sim|\bar{\tau}| /\left|\bar{\tau}_{c}\right| \sim \lambda^{-1 / 15} \gg 1\right)$. If we continue to use the asymptotic estimate Eq. (3.85c) beyond its formal range of validity we find that (for $d=3$ ) the barrier height is of order one at

$$
|\bar{\tau}|=\left|\bar{\tau}_{\text {cond }}\right| \sim \lambda^{(5-d) /(7-d)} \sim \lambda^{1 / 2},
$$

which represents a crude estimate of the condensation point. We also note from Eq. (3.87) that for $d=2$ the barrier height becomes of order unity precisely at $\bar{\tau}=\bar{\tau}_{G 1}$.

Let us compare our results with those of Fredrickson and Binder [10]. As mentioned in the Introduction these authors confined themselves to isotropic droplets, so we should compare with our Eq. (3.86). The scaled free energy (2.10) of Ref [10] is the same as our $\overline{\mathcal{F}}$, Eq. (2.1b), since all quantities are $O(1)$ except for the coupling constant

$$
u \sim \bar{N}^{-1 / 2} \sim \lambda \ll 1
$$

[here and below $\lambda$ refers to our coupling constant, not to their parameter $\lambda$, which is $O(1)$; note also that the quantity $\delta_{F B}$ defined in their Eq. (3.12) corresponds to our $\left.|\bar{\tau}|-\left|\bar{\tau}_{c}\right|=\delta\left|\bar{\tau}_{c}\right|\right]$. The estimate of the barrier in their Eq. (3.16) is $\bar{B}_{i s o} \sim \bar{N}^{-1 / 3}\left[\delta\left|\bar{\tau}_{c}\right|\right]^{-2} \sim \lambda^{-2 / 3} \delta^{-2}$, which agrees 
with Eq. (3.86a for $d=3$. However, the condition of validity of the expansion in their Eq. (3.14) is $\delta_{F B} \ll \bar{N}^{-1 / 3}$, not $\delta_{F B} \ll 1$ [i.e. $\delta \ll 1$, not $\delta \ll \lambda^{-2 / 3}$ ]. Thus the estimate of a kinetic limit of metastability or condensation point where $\bar{B}_{i s o} \sim 1$ given by these authors, namely $\delta_{F B} \sim \bar{N}^{-1 / 6} \sim \lambda^{1 / 3}$ (or $\left|\bar{\tau}_{\text {cond }}\right| \sim \lambda^{1 / 3}$ ) differs significantly from our estimate for the isotropic droplet $\left|\bar{\tau}_{\text {cond }}\right| \sim 1$ which follows from Eq. (3.860). In any case, according to our calculations the critical droplet is an anisotropic ramified structure, and it leads to the estimate Eq. (3.88) for the kinetic limit of metastability.

\section{Conclusion}

In this paper we have presented estimates of the critical droplet free energy of the Brazovskii model (2.1) in the metastable phase of its fluctuation induced first-order transition in the weak-coupling, low-noise limit $\lambda \ll 1$. Our work builds on that of Fredrickson and Binder [10], but finds a more favorable configuration for the critical nucleus than their isotropic droplet, by taking into account the anisotropy of the lamellar ordered state. Our derivation is also physically more plausible than that of Ref. [10] in our opinion, since the free energy we use distinguishes between the short-scale fluctuations leading to the first-order transition and the long-wavelength fluctuations necessary to build interfaces and droplets. In the final results, however, this added self-consistency changes only the numerical factors and not the basic scaling of the barrier height in the small parameter $\lambda$. Our main conclusion, which differs from that of Ref. [10] is that on the scale of the first order transition $\left[|\bar{\tau}|-\left|\bar{\tau}_{c}\right| \sim\left|\bar{\tau}_{c}\right|\right]$ the critical barrier is large, $\bar{B}_{W} \sim \lambda^{(1-d) / 6} \gg 1$, for $d \geq 2$.

At this point none of our calculations can be claimed to yield realistic estimates of the lifetimes of metastable states in the systems described by the Brazovskii free-energy $(2.1 \mathrm{~b})$. This is first of all because our results are based exclusively on dimensional analysis with no account of numerical coefficients, and with highly idealized asymptotic conditions, the most extreme of which is the one in Eq. (2.34). In addition, we have only addressed the question of finding the energy of the saddle-point configuration, which is the first step to estimating the lifetime of a metastable state, but by no means the whole answer.

Our work is to our knowledge the first attempt to modify classical nucleation theory $[11,12]$ in a controlled way to deal with metastability in fluctuation-driven first-order transitions [13]. A straightforward extension would be to solve the self-consistent equations (3.59) (3.61) for the Wulff droplet numerically and thereby calculate the function $\tilde{B}_{W}^{s c}(\delta)$ explicitly. A more difficult (and more interesting) step would be to justify the heuristic arguments leading to Eqs. (3.42) and (3.44) by a formal calculation analogous to Langer's [12] field-theoretic derivation of the lifetime of a metastable state in transitions where phase coexistence already appears at the mean-field level.

The most promising application to an experimental system is to the microphase separation transition in symmetric diblock copolymers [6-9], which was the main focus of the work of Fredrickson and Binder [10]. With all the caveats expressed above, we can say that we expect the disordered phase to be metastable against homogeneous nucleation of droplets down to a reduced temperature of the same order as or larger than the shift $\left|\bar{\tau}_{c}\right| \sim \lambda^{2 / 3}$ between the mean-field and the actual (thermodynamic) transition $\left(\left|\bar{\tau}_{\text {cond }}\right| \geq\left|\bar{\tau}_{c}\right|\right)$. If the ordered phase is homogeneously nucleated we expect the critical droplets to be ramified [16], since the more regular Wulff needles shown in Fig. 5b have large barriers. The actual shapes that would be observed experimentally can only be determined if one studies the subsequent evolution of the droplets as they aggregate and coarsen [10], a question whose elucidation requires further work.

Another possible experimental application is to Rayleigh-Bénard convection [4,5,27,28], perhaps near the fluid critical point where fluctuation effects are expected to be large [29]. In that case, 
however, apart from non-Boussinesq effects [27] which might mask the fluctuation contributions, it may be important to take into account the effects of multiplicative noise [30] on the transition, since the latter could be much larger than the additive (thermal) noise. It is not clear at present how much of the present theory would be relevant in the convection system.

Acknowledgements: One of us $(\mathrm{PCH})$ wishes to acknowledge numerous helpful discussions with David Huse. The research of JBS was supported in part by DOE under grant \#DE-FG0393ER14312. 


\section{Appendix A: Renormalization Group Recursion Relations}

We seek to obtain a coarse-grained free energy by constructing a momentum-shell renormalization group in the manner of Wilson and Kogut [22], except that we eliminate shells surrounding the sphere $|\mathbf{q}|=q_{0}$, rather than shells surrounding a single point in $q$-space, as was done in Ref. [22]. To do this we borrow from the techniques developed by Shankar [26] for the Fermi liquid. Rather than starting from the free-energy (2.1b), we generalize in the usual way [22] to include higher interactions, i.e. we start from

$$
\begin{gathered}
\overline{\mathcal{F}}[\bar{\psi}]=\overline{\mathcal{F}}_{2}+\overline{\mathcal{F}}_{4}+\overline{\mathcal{F}}_{6}+\ldots \\
\overline{\mathcal{F}}_{2}=\frac{1}{2} \int_{\left[0, \bar{\Lambda}_{0}\right]} d 1 d 2 \bar{u}_{2}(1,2)(2 \pi)^{d} \delta(1+2) \bar{\psi}(1) \bar{\psi}(2) \\
\overline{\mathcal{F}}_{4}=\frac{1}{4 !} \int_{\left[0, \bar{\Lambda}_{0}\right]} d 1 \ldots d 4 \bar{u}_{4}(1,2,3,4)(2 \pi)^{d} \delta(1+2+3+4) \bar{\psi}(1) \bar{\psi}(2) \bar{\psi}(3) \bar{\psi}(4),
\end{gathered}
$$

etc, where

$$
\int_{\left[0, \bar{\Lambda}_{0}\right]} \equiv \frac{1}{(2 \pi)^{d}} \int_{0<\left|\bar{q}_{1}-\bar{q}_{0}\right|<\bar{\Lambda}_{0}} d \bar{q}_{1} \bar{q}_{1}^{d-1} \int d \Omega_{1}
$$

and $d \Omega_{1}$ is the element of solid angle in $d$-dimensions. We now integrate over the modes

$$
\bar{\Lambda}_{0} / b<\bar{q}-\bar{q}_{0}<\bar{\Lambda}_{0}
$$

and the corresponding inner shell with $\bar{q}<\bar{q}_{0}$, and then rescale all momenta so that $\bar{\Lambda}_{0} / b \rightarrow \bar{\Lambda}_{0}$. If we define

$$
\begin{aligned}
\bar{k} & =\bar{q}-\bar{q}_{0}, \\
\bar{k}^{\prime} & =b \bar{k} \\
\bar{\psi}^{\prime}\left(\bar{k}^{\prime}\right) & =b^{-3 / 2} \bar{\psi}(\bar{k}),
\end{aligned}
$$

then in lowest order in $\bar{u}_{4} \sim \lambda$ we may write

$$
\bar{u}_{2}(1,2)=\left[\bar{r}+\tilde{\xi}_{0}^{4}\left(\bar{q}_{1}^{2}-\bar{q}_{0}^{2}\right)^{2}\right]
$$

and the change in $\bar{r}$ is given by

$$
\bar{r}^{\prime}=b^{2}\left[\bar{r}+\Delta_{2}\right],
$$

where $\Delta_{2}$ is the contribution from the Hartree diagram in Fig. 1a (see below). Let us now examine the change in $\bar{u}_{4}$. By an argument similar to the one given by Shankar [26] (Sec. V) we find that at "tree-level" the change in $\bar{u}_{4}$ is given by

$$
\bar{u}_{4}^{\prime}\left(1^{\prime}, 2^{\prime}, 3^{\prime}, 4^{\prime}\right)=e^{-(b-1) \bar{q}_{0}\left[\left|\hat{\mathbf{n}}_{1}+\hat{\mathbf{n}}_{2}+\hat{\mathbf{n}}_{3}\right|-1\right] / \bar{\Lambda}_{0}} b^{3} \bar{u}_{4}(1,2,3,4),
$$

where $\hat{\mathbf{n}}_{i}$ is the unit vector $\overline{\mathbf{q}}_{i} /\left|\overline{\mathbf{q}}_{i}\right|$. Thus by iteration of the renormalization group, only couplings with vectors satisfying

$$
\left|\hat{\mathbf{n}}_{1}+\hat{\mathbf{n}}_{2}+\hat{\mathbf{n}}_{3}\right|=1
$$


will remain finite. It can be shown that for both $d=2$ and $d=3$ this implies that the four wavevectors in $\bar{u}_{4}(1,2,3,4)$ must be equal and opposite in pairs when their magnitude goes to $\bar{q}_{0}$. Thus only

$$
\bar{u}_{4}\left(\hat{\mathbf{n}}_{1},-\hat{\mathbf{n}}_{1}, \hat{\mathbf{n}}_{2},-\hat{\mathbf{n}}_{2} ; \bar{q}_{0}\right)=\bar{u}\left(\hat{\mathbf{n}}_{1}, \hat{\mathbf{n}}_{2}\right)
$$

survives, and it satisfies

$$
\bar{u}^{\prime}\left(\hat{\mathbf{n}}_{1}, \hat{\mathbf{n}}_{2}\right)=b^{3}\left[\bar{u}\left(\hat{\mathbf{n}}_{1}, \hat{\mathbf{n}}_{2}\right)+\Delta_{4}\left(\hat{\mathbf{n}}_{2}, \hat{\mathbf{n}}_{2}\right)\right],
$$

where $\Delta_{4}$ is the contribution from the diagrams in Figs. 1c and 1d. It turns out that $\bar{u}\left(\hat{\mathbf{n}}_{1}, \hat{\mathbf{n}}_{2}\right)$ develops a dependence on the angle between $\hat{\mathbf{n}}_{1}$ and $\hat{\mathbf{n}}_{2}$ under the action of the renormalization group. For a general nonzero angle $\bar{u}$ is practically constant and we define

$$
\bar{u}\left(\hat{\mathbf{n}}_{1}, \hat{\mathbf{n}}_{2}\right)=\bar{u}_{a} .
$$

For the special case of parallel $\hat{\mathbf{n}}_{1}$ and $\hat{\mathbf{n}}_{2}$ (to within an angle of $O(\sqrt{\bar{r}})$, we define

$$
\bar{u}\left(\hat{\mathbf{n}}_{1}, \hat{\mathbf{n}}_{1}\right)=\bar{u}_{b} .
$$

Similarly, we have

$$
\begin{gathered}
\bar{u}_{6}(1, \ldots, 6) \rightarrow \bar{u}_{6}\left(\hat{\mathbf{n}}_{1},-\hat{\mathbf{n}}_{1}, \hat{\mathbf{n}}_{2},-\hat{\mathbf{n}}_{2}, \hat{\mathbf{n}}_{3},-\hat{\mathbf{n}}_{3} ; \bar{q}_{0}\right)=\bar{w}\left(\hat{\mathbf{n}}_{1}, \hat{\mathbf{n}}_{2}, \hat{\mathbf{n}}_{3}\right), \\
\bar{w}^{\prime}\left(\hat{\mathbf{n}}_{1}, \hat{\mathbf{n}}_{2}, \hat{\mathbf{n}}_{3}\right)=b^{4}\left[\bar{w}\left(\hat{\mathbf{n}}_{1}, \hat{\mathbf{n}}_{2}, \hat{\mathbf{n}}_{3}\right)+\Delta_{6}\left(\hat{\mathbf{n}}_{1}, \hat{\mathbf{n}}_{2}, \hat{\mathbf{n}}_{3}\right)\right],
\end{gathered}
$$

with $\Delta_{6}$ given in Figs. 1e and 1f. Under the action of the renormalization group $\bar{w}$ also develops an angular dependence; for general angles between $\hat{\mathbf{n}}_{1}, \hat{\mathbf{n}}_{2}$ and $\hat{\mathbf{n}}_{3}$ we define

$$
\bar{w}=\bar{w}_{a} .
$$

When only two of the unit vectors are parallel we define

$$
\bar{w}=\bar{w}_{b},
$$

and for all three unit vectors parallel we define

$$
\bar{w}=\bar{w}_{c} .
$$

Let us now evaluate the contributions $\Delta_{2}, \Delta_{4}, \Delta_{6}$, using the lowest relevant order in $\bar{u}_{4} \sim \bar{u} \sim \lambda$ for each $\Delta_{i}$. We find

$$
\begin{aligned}
\Delta_{2} & =(2 \pi)^{-d} \int_{\bar{q}_{0}+\bar{\Lambda}_{0} / b}^{\bar{q}_{0}+\bar{\Lambda}_{0}} d \bar{q} \bar{q}_{0}^{d-1} \int d \Omega_{1} \frac{\bar{u}_{4}\left(\hat{\mathbf{n}},-\hat{\mathbf{n}}, \hat{\mathbf{n}}_{1},-\hat{\mathbf{n}}_{1}\right)}{\bar{r}+\tilde{\xi}_{0}^{4}\left(\bar{q}^{2}-\bar{q}_{0}^{2}\right)^{2}} \\
& =\bar{\alpha} \bar{\xi}_{0} \bar{u}_{a} \int_{\bar{\Lambda}_{0} / b}^{\bar{\Lambda}_{0}} d \bar{k} \frac{1}{\bar{r}+\bar{\xi}_{0}^{2} \bar{k}^{2}},
\end{aligned}
$$

where $\bar{\alpha}$ is given in Eq. (2.12) and $\bar{\xi}_{0}$ is defined in Eq. (2.15h). In particular there is no momentum dependence to this order, so the second term in square brackets in Eq. (A.6) is unmodified. In order to find differential recursion relations we set

$$
b=1+\ell,
$$


with $\ell \ll 1$, and find from Eqs. (A.7) and (A.19)

$$
\frac{d \bar{r}}{d \ell}=2 \bar{r}+\frac{\bar{\alpha} \bar{\xi}_{0} \bar{u}_{a} \bar{\Lambda}_{0}}{\bar{r}+\bar{\xi}_{0}^{2} \bar{\Lambda}_{0}^{2}}
$$

The contributions to $\Delta_{4}$ are given in Figs. $1 \mathrm{c}$ and $1 \mathrm{~d}$. When we examine the contribution to $\bar{u}_{a}$ from Fig. $1 \mathrm{~b}$ we find there is one channel in which both intermediate Green's functions have arguments in the shell being integrated over, whereas for $\bar{u}_{b}$ there are two. This allows the evaluation of $\Delta_{4}$ and leads to

$$
\frac{d \bar{u}_{a}}{d \ell}=3 \bar{u}_{a}-\frac{\bar{\alpha} \bar{\xi}_{0} \bar{u}_{a}^{2} \bar{\Lambda}_{0}}{\left(\bar{r}+\bar{\xi}_{0}^{2} \bar{\Lambda}_{0}^{2}\right)^{2}}+\frac{\bar{\alpha} \bar{\xi}_{0} \bar{w}_{a} \bar{\Lambda}_{0}}{\bar{r}+\bar{\xi}_{0}^{2} \bar{\Lambda}_{0}^{2}}
$$

and

$$
\frac{d \bar{u}_{b}}{d \ell}=3 \bar{u}_{b}-\frac{2 \bar{\alpha} \bar{\xi}_{0} \bar{u}_{a}^{2} \bar{\Lambda}_{0}}{\left(\bar{r}+\bar{\xi}_{0}^{2} \bar{\Lambda}_{0}^{2}\right)^{2}}+\frac{\bar{\alpha} \bar{\xi}_{0} \bar{w}_{b} \bar{\Lambda}_{0}}{\bar{r}+\bar{\xi}_{0}^{2} \bar{\Lambda}_{0}^{2}} .
$$

Now consider $\Delta_{6}$. There are 15 ways of distributing the 6 arguments of $\bar{u}_{6}$ over the external legs in both Figs. 1e and 1f. Examination of the number of channels which contribute to $\bar{w}_{a}, \bar{w}_{b}$ and $\bar{w}_{c}$ leads to

$$
\frac{d \bar{w}_{a}}{d \ell}=4 \bar{w}_{a}+\frac{2 \bar{\alpha} \bar{\xi}_{0} \bar{u}_{a}^{3} \bar{\Lambda}_{0}}{\left(\bar{r}+\bar{\xi}_{0}^{2} \bar{\Lambda}_{0}^{2}\right)^{3}}-\frac{3 \bar{\alpha} \bar{\xi}_{0} \bar{u}_{a} \bar{w}_{a} \bar{\Lambda}_{0}}{\left(\bar{r}+\bar{\xi}_{0}^{2} \bar{\Lambda}_{0}^{2}\right)^{2}},
$$

and two similar equations for $\bar{w}_{b}$ and $\bar{w}_{c}$.

These equations have initial values corresponding to Eq. (2.1b),

$$
\begin{aligned}
& \bar{r}(\ell=0)=\bar{\tau} \\
& \bar{u}_{a}(\ell=0)=\bar{u}_{b}(\ell=0)=\lambda, \\
& \bar{w}_{a}(\ell=0)=\bar{w}_{b}(\ell=0)=\bar{w}_{c}(\ell=0)=0 .
\end{aligned}
$$

Because of the scalings we have chosen in Eq. (A.5) the quantities $\bar{r}(\ell), \bar{u}_{a, b}(\ell), \bar{w}_{a, b, c}(\ell)$ all grow with $\ell$. To find finite quantities we make a change of variables

$$
\begin{aligned}
\Lambda & =\bar{\Lambda}_{0} \bar{\xi}_{0}(\bar{\alpha} \lambda)^{-1 / 3} e^{-\ell}, \\
r(\Lambda) & =\bar{r}(\ell)(\bar{\alpha} \lambda)^{-2 / 3} e^{-2 \ell}, \\
u_{a, b}(\Lambda) & =\bar{u}_{a, b}(\ell) \lambda e^{-3 \ell}, \\
w_{a, b, c}(\Lambda) & =\bar{w}_{a, b, c}(\ell) \bar{\alpha}^{2}(\bar{\alpha} \lambda)^{-4 / 3} e^{-4 \ell},
\end{aligned}
$$

leading to

$$
\begin{aligned}
\frac{d r}{d \Lambda} & =-\frac{u_{a}}{r+\Lambda^{2}}, \\
\frac{d u_{a}}{d \Lambda} & =\frac{u_{a}^{2}}{\left(r+\Lambda^{2}\right)^{2}}-\frac{w_{a}}{r+\Lambda}, \\
\frac{d u_{b}}{d \Lambda} & =\frac{2 u_{a}^{2}}{\left(r+\Lambda^{2}\right)^{2}}-\frac{w_{b}}{r+\Lambda^{2}},
\end{aligned}
$$




$$
\begin{aligned}
\frac{d w_{a}}{d \Lambda} & =\frac{-2 u_{a}^{3}}{\left(r+\Lambda^{2}\right)^{3}}+\frac{3 u_{a} w_{a}}{\left(r+\Lambda^{2}\right)^{2}}, \\
\frac{d w_{b}}{d \Lambda} & =\frac{-4 u_{a}^{3}}{\left(r+\Lambda^{2}\right)^{3}}+\frac{u_{a} w_{b}}{\left(r+\Lambda^{2}\right)^{2}}+\frac{4 u_{a} w_{a}}{\left(r+\Lambda^{2}\right)^{2}}, \\
\frac{d w_{c}}{d \Lambda} & =\frac{-12 u_{a}^{3}}{\left(r+\Lambda^{2}\right)^{3}}+\frac{8 u_{a} w_{b}}{\left(r+\Lambda^{2}\right)^{2}},
\end{aligned}
$$

with initial values at $\Lambda=\bar{\Lambda}_{0} \bar{\xi}_{0}(\bar{\alpha} \lambda)^{-1 / 3}=\infty$,

$$
r(\infty)=\tau, \quad u_{a, b}(\infty)=1, \quad w_{a, b, c}(\infty)=0 .
$$

Let us reexpress the free energy in terms of the running couplings $\bar{u}_{n}(\ell)$. Since the upper cutoff $\bar{\Lambda}_{0}$ is restored to its original value at each stage of the recursion (A.7), the effective free energy still has the form (A.1-2) when expressed in terms of the $\bar{u}_{n}(\ell)$ and the corresponding $\bar{\psi}(\ell)$. Let us now change to the scaling (A.23), supplemented with the relation

$$
\psi(\ell)=\bar{\psi}(\ell) \lambda^{1 / 2}(\bar{\alpha} \lambda)^{-1 / 3} e^{3 \ell / 2} .
$$

Then it is straightforward to show that the free energy takes precisely the form (2.44), with a variable upper cutoff $\Lambda$, and coefficients satisfying Eqs. (A.24). The resulting functions $r(\Lambda), u(\Lambda)=$ $u_{b}(\Lambda), w(\Lambda)=w_{c}(\Lambda)$ are similar, but not identical to the ones defined in Eqs. (2.45-48) and obtained phenomenologically in Appendix B. In particular, contrary to the latter functions, the solutions of (A.24) do not exist for all $\tau$ and $\Lambda$, due to singular denominators for $\tau<\tau_{1}=-2.66$. This is to be contrasted with the bulk transition predicted by (A.24) at $\tau_{c}^{r g}=-2.56$. An analysis of Eqs. (A.24) shows that the coefficients are undefined for

$$
\Lambda<\Lambda_{1}(\tau)
$$

where $\Lambda_{1}(\tau)$ is plotted in Fig. 11. In the domain (A.27) represented by the shaded region of Fig. 11, the quantity $r(\Lambda, \tau)+\Lambda^{2}$ appearing in the denominators in (A.24) has zeroes, so the recursion relations cannot be solved with initial conditions at $\Lambda=\infty$. Of course the singularities in (A.24) occur for $r(\Lambda, \tau)<0$, i.e. in a region where the disordered state is not even metastable, so they are not physically significant. However, in the renormalization group formulation the coarsegrained free energy at small $\Lambda$ is found by integration starting from a bare free energy with large $\Lambda$, so the recursion relations are needed even in the unphysical parameter range with $\tau<\tau_{c}, \Lambda$ large, and $r<0$. The phenomenological coarse-graining procedure of Eqs. (2.45-48) avoids the singularity, but even if it did not, this would not affect the utility of the method. This is because the phenomenological equations are given in integrated form, so the coefficients $r(\Lambda), u(\Lambda), w(\Lambda)$ at a particular value of $\Lambda$ are obtained by solving equations such as (2.45-48) involving only the same value of $\Lambda$, rather than by integrating down from large $\Lambda$. This means that the physically relevant small- $\Lambda$ behavior can be obtained independently of any large- $\Lambda$ singularities.

In Fig. 12 we show the numerical solution of the recursion relations (A.24) in a form similar to that in Fig. 2. The results only exist to the right of the shaded region in Fig. 11, i.e. for $\tau>\tau_{1}$ when $\Lambda=0$. The coefficients agree with the phenomenological ones for $\tau \gtrsim 0$, but they differ quantitatively for $\tau<0$ due to the vanishing of $r$ at $\tau_{1}$. Nevertheless, for $\tau>\tau_{1}$ the recursion relations provide a justification for the phenomenological theory, since they have been derived by more or less standard diagrammatic methods. 


\section{Appendix B: Phenomenological Coarse-graining Procedure}

In this Appendix we reformulate the derivation of Fredrickson and Binder [10] for the effective free energy, replacing the averages in their Eq. (A.3), which are over the whole range $0<\left|q-q_{0}\right|<\Lambda_{0}$, by averages over the restricted range $0<\left|q-q_{0}\right|<\Lambda$. The coefficients $\bar{r}(\Lambda), \bar{u}(\Lambda), \bar{w}(\Lambda)$ are just the derivatives

$$
\Gamma_{\Lambda n}=\frac{\delta^{n} \overline{\mathcal{F}}_{\Lambda}}{\delta \bar{\psi}^{n}}
$$

for $n=2,4,6$, respectively. They are obtained from the diagrams in Fig. 1 except that the integrals are confined to the range $0<\left|q-q_{0}\right|<\Lambda$, and no rescaling of the momentum is performed. As we noted in our discussion of the renormalization group in Appendix A, the number of channels which contribute to $\Gamma_{4}$ and $\Gamma_{6}$ depends on the angles between the wavevectors which are their arguments. We will eventually want the free energy to be a functional of a $\psi$ of the form (3.3). Thus we want the wavevectors occurring in the arguments to be either parallel or antiparallel. In the notation of Appendix A we then want to identify $u_{b}$ with $u$ and $w_{c}$ with $w$.

For $r$ the Hartree graph in Fig. 1a gives

$$
\bar{r}(\Lambda)=\bar{\tau}+\bar{\alpha} \bar{\xi}_{0} \lambda \int_{\bar{\Lambda}}^{\bar{\Lambda}_{0}} \frac{d \bar{k}}{\bar{r}+\bar{\xi}_{0}^{2} \bar{k}^{2}} .
$$

For $\bar{u}_{a}$ the graph in Fig. 1c yields

$$
\bar{u}_{a}=\lambda-\bar{u}_{a} \lambda \int_{\bar{\Lambda}}^{\bar{\Lambda}_{0}} \bar{\alpha} \bar{\xi}_{0} \frac{d \bar{k}}{\left(\bar{r}+\bar{\xi}_{0}^{2} \bar{k}^{2}\right)^{2}} .
$$

For $\bar{u}_{b}$ this graph yields

$$
\bar{u}_{b}=\lambda-2 \bar{u}_{a} \lambda \int_{\bar{\Lambda}}^{\bar{\Lambda}_{0}} \bar{\alpha} \bar{\xi}_{0} \frac{d \bar{k}}{\left(\bar{r}+\bar{\xi}_{0}^{2} \bar{k}^{2}\right)^{2}} .
$$

The coefficient of the sixth order term, $\bar{w}_{c}$, is given by the graph in Fig. 1e which yields

$$
\bar{w}_{c}=12 \int_{\bar{\Lambda}}^{\bar{\Lambda}_{0}} \bar{u}_{a}^{3} \bar{\alpha} \bar{\xi}_{0} \frac{d k}{\left(\bar{r}+\bar{\xi}_{0}^{2} \bar{k}^{2}\right)^{3}} .
$$

When Eqs. (B.2-B.5) are reexpressed in terms of the scaled units of Eq. (2.15) they then yield precisely Eqs. (2.45-48).

Let us verify that the denominator in the $\phi_{n}$, Eq (2.48) remains positive

$$
r(\Lambda, \tau)+\Lambda^{2}>0,
$$

from which it follows that the coefficients $r(\Lambda, \tau), u(\Lambda, \tau)$ and $w(\Lambda, \tau)$, Eqs. (2.45-48) are welldefined for all $\Lambda$ and $\tau$. The question only arises for $r<0$ and $\tau<0$, so we set

$$
r=-\Lambda^{2}(1-\eta)^{2}
$$

and ask whether $\eta$ can vanish. If we carry out the integral $\phi_{1}$ in Eq. (2.48) we find (assuming $\eta>0)$

$$
r=-\Lambda^{2}(1-\eta)^{2}=\tau+\frac{1}{2 \Lambda(1-\eta)} \ln \left(\frac{2-\eta}{\eta}\right)
$$

which for $\eta \rightarrow 0$ becomes

$$
\ln \frac{2}{\eta}=2 \Lambda\left(|\tau|-\Lambda^{2}\right)
$$

Since for fixed $\tau$ the rhs of $(\overline{\mathrm{B} .9})$ is bounded by $4(|\tau| / 3)^{3 / 2}$ as a function of $\Lambda$, we conclude that (B.9) has no solution for $\eta \rightarrow 0$, and that the inequality (B.6) is always satisfied. 


\section{References}

1. S. A. Brazovskii, Sov. Phys. JETP 41, 85 (1975).

2. D. Briskin, D. L. Johnson, H. Fellner, and M. E. Neubert, Phys. Rev. Let. 50, 178 (1983); L. J. Martinez-Miranda, A. R. Kortan, and R. J. Birgeneau, ibid 56, 2264 (1986); C. W. Garland and M. E. Huster, Phys. Rev. A35, 2365 (1987).

3. R. F. Sawyer, Phys. Rev. Lett. 29, 382 (1972); A. N. Dyugaev, JETP Lett. 22, 83 (1975).

4. J. B. Swift and P. C. Hohenberg, Phys. Rev. A15, 319 (1977).

5. P. C. Hohenberg and J. B. Swift, Phys. Rev. A46, 4773 (1992).

6. L. Leibler, Macromolecules 13, 1602 (1980).

7. G. H. Fredrickson and E. Helfand, J. Chem. Phys. 87, 697 (1987).

8. F. S. Bates, J. H. Rosedale, G. H. Fredrickson and C. J. Glinka, Phys. Rev. Lett. 61, 2229 (1988).

9. F. S. Bates, J. H. Rosedale, and G. H. Fredrickson, J. Chem. Phys. 92, 6225 (1990).

10. G. H. Fredrickson and K. Binder, J. Chem. Phys. 91, 7265 (1989).

11. F. F. Abraham, Homogeneous Nucleation Theory (Academic, NY, 1974); J. D. Gunton, M. San Miguel, and P. S. Sahni, in Phase Transitions and Critical Phenomena, edited by C. Domb and J. L. Lebowitz (Academic, NY, 1983) Vol. 8; J. D. Gunton and M. Droz, Introduction to the Theory of Metastable and Unstable States, Lecture Notes in Physics 183 (Springer Verlag, Berlin, 1983).

12. J. S. Langer, Ann. Phys. (NY) 41, 108 (1967); 54, 258 (1969); in Systems Far from Equilibrium, Lecture Notes in Physics 132 (Springer Verlag, Berlin, 1980).

13. J. Rudnick, Phys. Rev. B18, 1406 (1978) and references therein.

14. See L. D. Landau and E. M. Lifshitz, Statistical Physics (Addison-Wesley, London, 1958) Ch. $\mathrm{XV}$.

15. J. B. Fournier and G. Durand, J. Phys. II France 1, 845 (1991).

16. C. Unger and W. Klein, Phys. Rev. B29, 2698 (1984).

17. P. C. Hohenberg and B. I. Halperin, Rev. Mod. Phys. 49, 435 (1977).

18. V. L. Ginzburg, Sov. Phys. Sol. State 2, 1824 (1960).

19. For greater clarity of presentation we have given all our definitions in this subsection in terms of the unbarred (scaled) quantities $q, \Lambda, \psi, \mathcal{F}$, etc of Eqs. (2.15), but analogous definitions would hold for the original barred quantities. In Appendix A we define a different coarse graining procedure in terms of quantities $\bar{r}(\bar{\Lambda}), \bar{u}(\bar{\Lambda}), \bar{w}(\bar{\Lambda})$ which are not obtained from $r(\Lambda)$, $u(\Lambda) w(\Lambda)$ via Eq. (2.15). 
20. Since $\Lambda_{0}=q_{0}$, and according to Eq. (2.15) $q_{0}=\left(2 \tilde{\xi}_{0}^{2}\right)^{-1}(\alpha \lambda)^{-1 / 3} \gg 1$, for $\lambda \ll 1$, we will henceforth set $\Lambda_{0}=\infty$, as in the upper limit of the integral of Eq. (2.48).

21. In this paper we refer to the function $\overline{\mathcal{F}}$ in the starting Brazovskii model (2.1b) as a "free energy" since it is obtained by coarse-graining a more microscopic Hamiltonian. Subsequent coarse-grained versions, denoted $\mathcal{F}_{\Lambda}$ are also referred to as "free energies". From the point of view of nucleation theory, on the other hand, since we are only discussing saddle-point configurations, the quantities we calculate are "energy barriers". In order to estimate "freeenergy barriers", we would have to take into account the "entropic" contributions to the lifetime of the metastable state coming from other configurations, for example the small oscillations around the saddle. Since we neglect these effects in the present work we use the terms energy and free energy somewhat loosely.

22. K. G. Wilson and J. B. Kogut, Phys. Rept. 12, 7 (1974).

23. S. K. Ma, Modern Theory of Critical Phenomena (Benjamin-Cummings, Reading, MA, 1976).

24. D. Mukamel and R. M. Hornreich, J. Phys. C13, 161 (1980).

25. D. D. Ling, B. Friman, and G. Grinstein, Phys. Rev. B24, 2718 (1981).

26. R. Shankar, Rev. Mod. Phys. 66, 129 (1994).

27. See M. C. Cross and P. C. Hohenberg, Rev. Mod. Phys. 65, 851 (1993), Sec. IV.A.1.

28. M. C. Cross, Phys. Rev. A25, 1065 (1982).

29. V. Steinberg and S. A. Brazovskii, (unpublished).

30. M. V. Feigelman and I. E. Staroselsky, Z. Phys. B. Cond. Mat. 62, 261 (1986). 


\section{Figure Captions}

1. Low-order diagrams in the weak-coupling expansion of the Brazovskii model (2.1b).

(a) Hartree diagram contributing to $r$, or $\Delta_{2}$.

(b) The second-order self-energy diagram entering $\Sigma_{2}$ in Eq. (2.29).

(c) A diagram contributing to $\Delta_{4}$. The three ways of distributing the arguments of $u_{4}(1,2,3,4)$ are shown.

(d) Another diagram contributing to $\Delta_{4}$.

(e) A diagram contributing to $\Delta_{6}$. The fifteen ways of distributing the arguments of $u_{6}(1,2,3,4,5,6)$ are not shown.

(f) Another diagram contributing to $\Delta_{6}$.

2. The coefficients of the bulk free energy (2.23), plotted as a function of $\tau$. (a) The coefficient $r$, obtained by solving Eq. (2.19). (b) The coefficient $u$, from Eq. (2.24). (c) The coefficient $w$, from Eq. (2.25). The bulk coefficients correspond to the limit $\Lambda=0$ of the coefficients of Eqs. (2.45-48).

3. Schematic diagram of a longitudinal interface between the lamellar $(x>0)$ and disordered state $(x<0)$. (a) Sketch of the lamellae; (b) Order parameter $|A|$ vs. $x$. The width of the interface is $\xi_{\|}$.

4. As in the preceding figure, but for a transverse interface, with lamellae for $y>0$ and a disordered state for $y<0$. The transverse interface is thinner than the longitudinal one $\left(\xi_{\perp} \ll \xi_{\|}\right)$.

5. Schematic diagram of critical droplets. (a) Isotropic droplet with concentric lamellae. (b) The anisotropic Wulff droplet, consisting of lamellae perpendicular to $x$.

6. The parallelipiped entering the Wulff construction.

7. A portion of the Brazovskii sphere in reciprocal space, showing the relationship between the longitudinal cutoff $\Lambda$ and the transverse cutoff $\left(2 q_{0} \Lambda\right)^{1 / 2} \gg \Lambda$, for $\Lambda \ll q_{0}$.

8. Schematic representation of focal conic defects. (a) and (b) represent a single focal conic introduced into the Wulff droplet; (c) is a biconical domain in which focal conics are juxtaposed; (d) is a focal conic spherical network of cones representing the equilibrium shape for arbitrarily large droplets. [From Ref. 15.]

9. Possible distortions of the Wulff droplet to eliminate longitudinal interfaces. (a) An annular shape with no defects; (b) the same, but with the bend relieved by the introduction of dislocations.

10. A summary of the metastability properties of the Brazovskii model in three dimensions, for $\lambda \ll 1$. Shown are both the scale of the control parameter $\bar{\tau}$ of Eq. (2.1b), and of the reduced quantity $\tau \sim \bar{\tau} \lambda^{-2 / 3}$. The fluctuation induced bulk transition occurs for $\tau<0$, at $\tau=\tau_{c}=O(1)\left[\bar{\tau}=\bar{\tau}_{c}=O\left(\lambda^{2 / 3}\right)\right]$. The defected critical droplets are only favorable in an infinitesimal region near $\tau_{c}, \delta=\left[|\tau|-\left|\tau_{c}\right|\right] /\left|\tau_{c}\right| \sim \lambda^{2 / 9} \ll 1$. The anisotropic Wulff droplet is favorable for $1>\delta>\lambda^{2 / 9}$. For $|\tau| \gg\left|\tau_{c}\right|$, [| $\left.|\bar{\tau}| \gg\left|\bar{\tau}_{c}\right|\right]$ the Wulff droplet is ramified 
since the interface width is of the same order as the droplet radius. The Ginzburg criterion beyond which the perturbation theory no longer holds occurs for $|\tau|=\left|\tau_{G 1}\right| \sim \lambda^{-1 / 15} \gg\left|\tau_{c}\right|$, $\left[|\bar{\tau}|=\left|\bar{\tau}_{G 1}\right|=\lambda^{3 / 5} \gg\left|\bar{\tau}_{c}\right|\right]$, at which point the critical barrier height is $\bar{B}_{G 1} \sim \lambda^{-1 / 5} \gg 1$. The barrier becomes of order unity at $|\tau|=\left|\tau_{\text {cond }}\right| \sim \lambda^{-1 / 6},\left[|\bar{\tau}|=\left|\bar{\tau}_{\text {cond }}\right| \sim \lambda^{1 / 2}\right]$.

11. The function $\Lambda_{1}(\tau)$ at which the recursion relations (A.24) have a singularity. The coefficients are well-defined for all $\Lambda$ if $\tau>\tau_{1}$ and for $\Lambda>\Lambda_{1}(\tau)$ if $\tau<\tau_{1}$.

12. The bulk coefficients obtained from the recursion relations (A.24). (a) The coefficient $r(\Lambda=$ $0, \tau)$ vs. $\tau$; (b) $u(\tau)=u_{b}(\Lambda=0, \tau)$ vs. $\tau$; (c) $w(\tau)=w_{c}(\Lambda=0, \tau)$ vs. $\tau$. The coefficients are only defined for $\tau>\tau_{1}=-2.65$. These results are to be compared with those in Fig. 2 . 

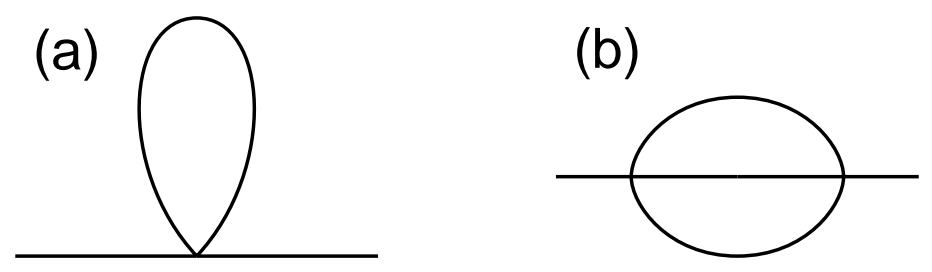

(c)

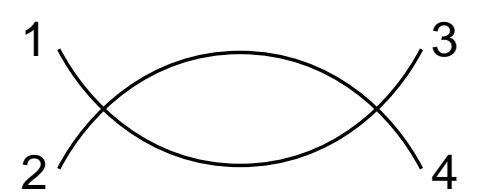

(d)

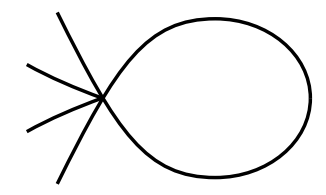

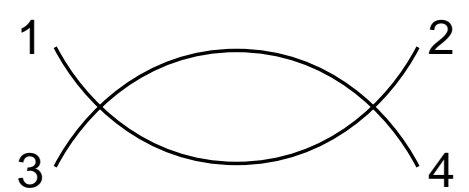

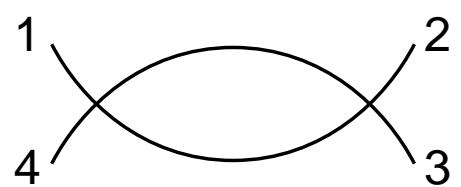

(e)

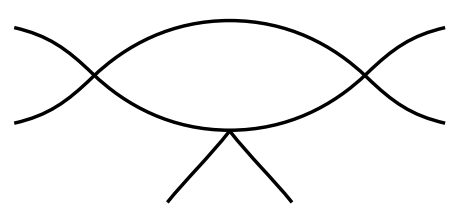

(f)

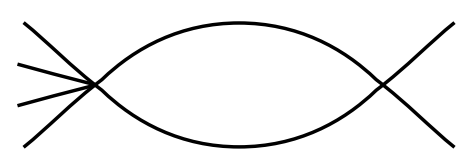




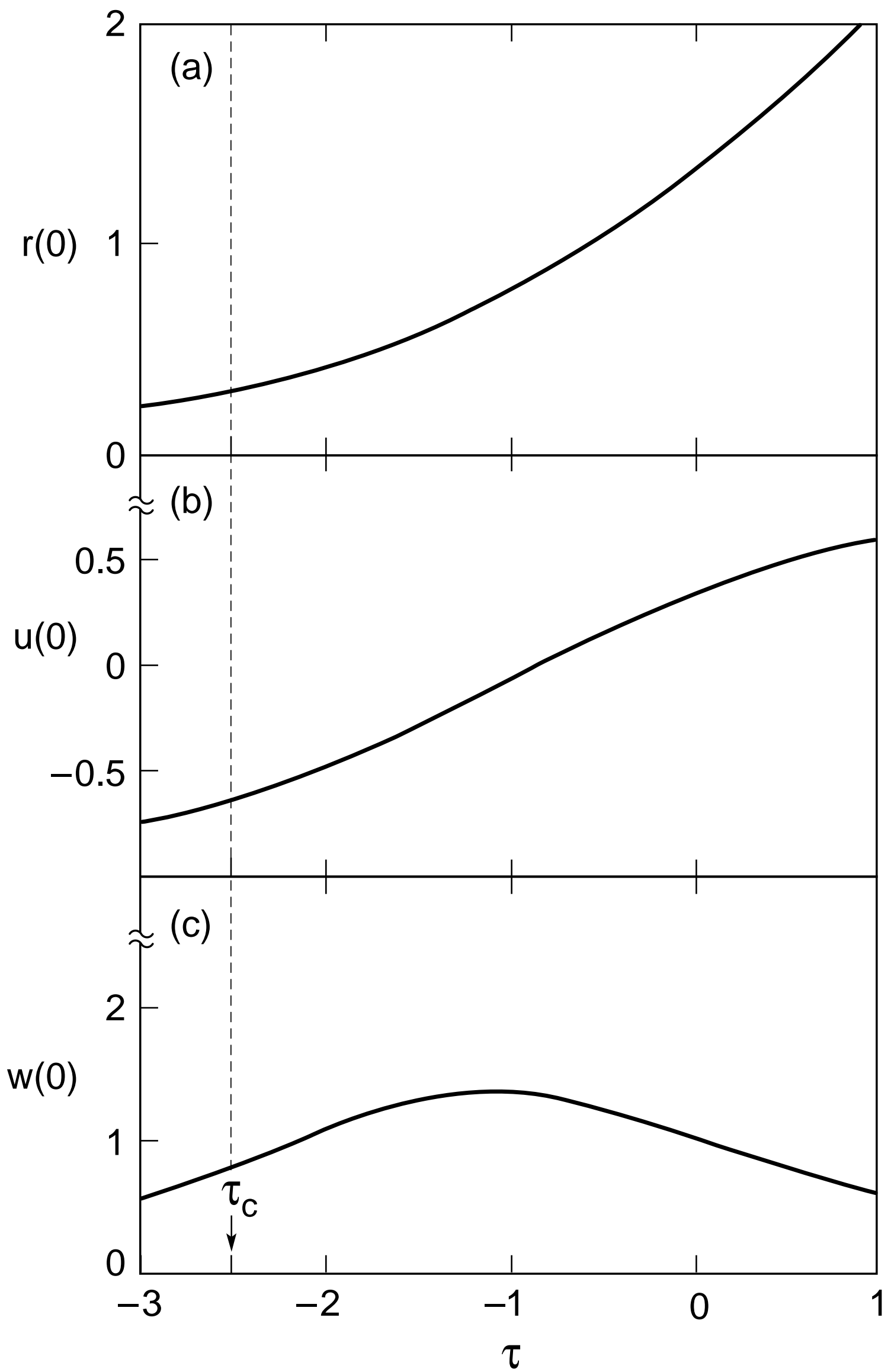




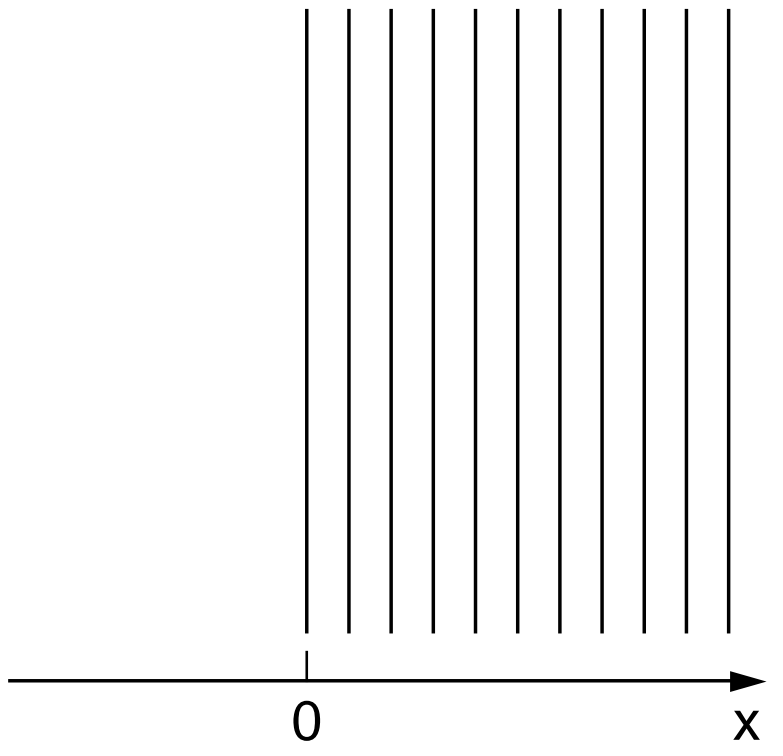

(a)

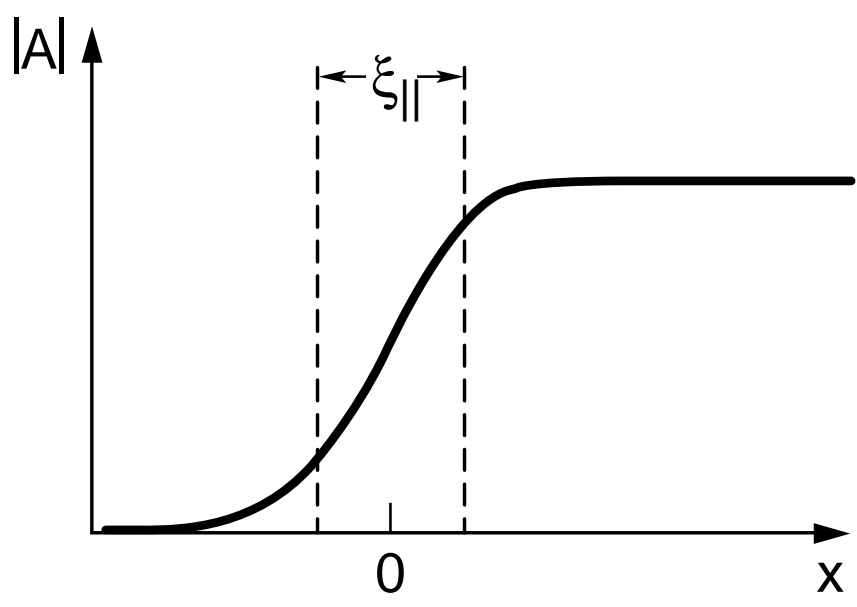

(b) 


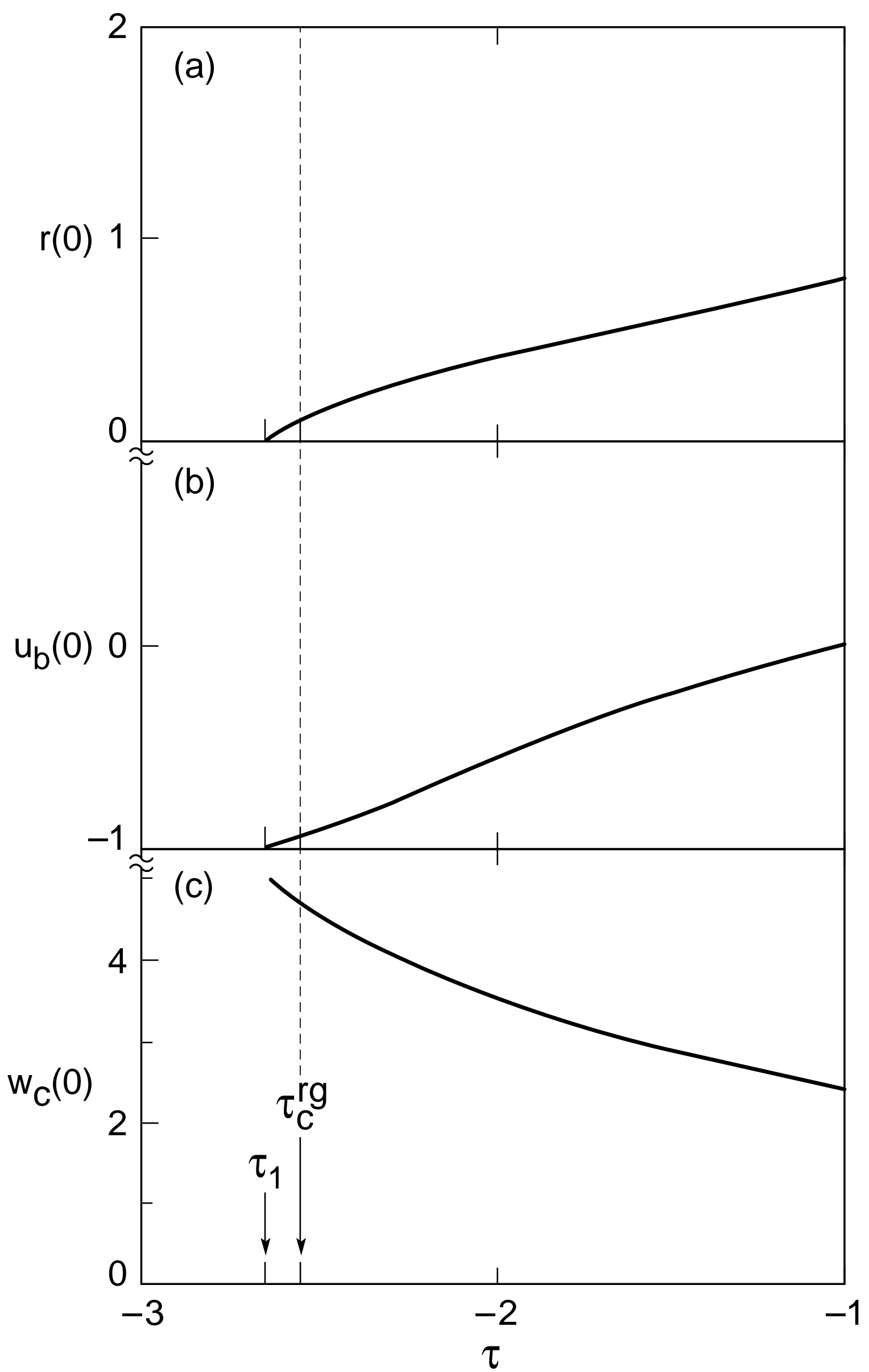




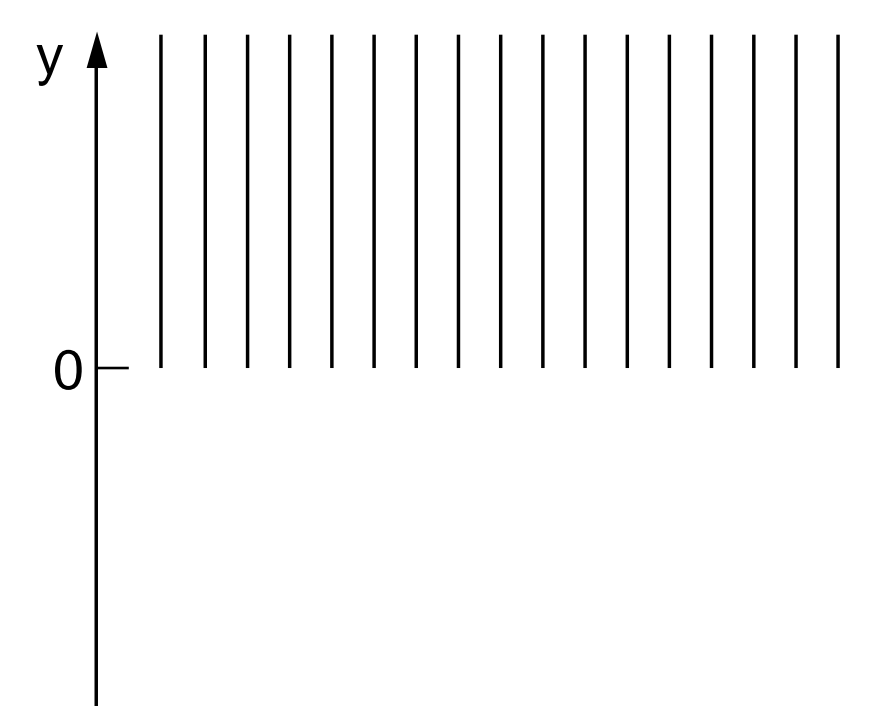

(a)

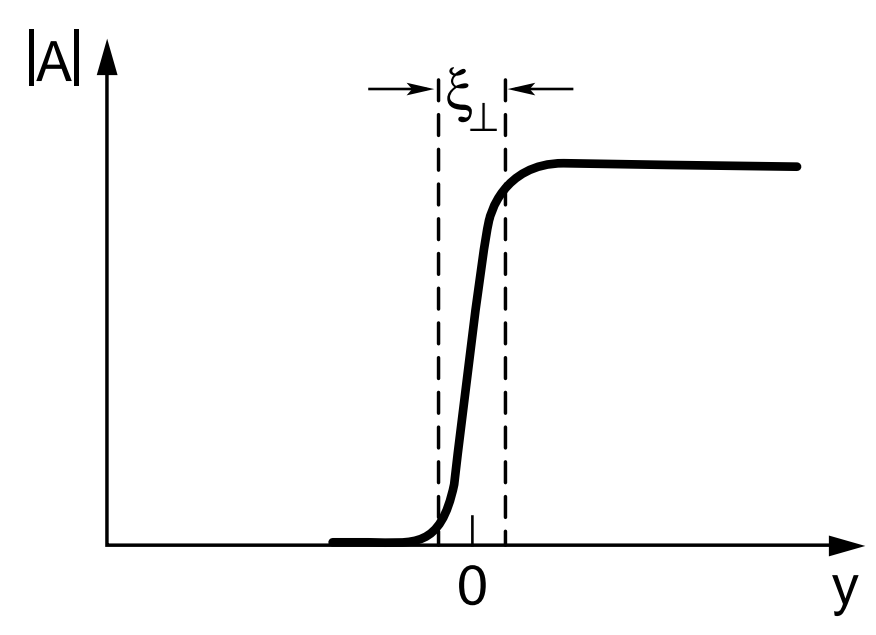

(b) 


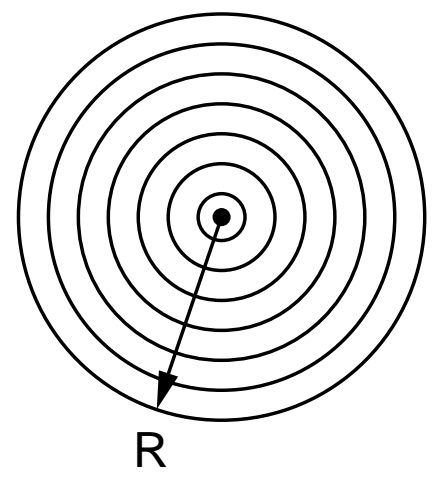

(a)

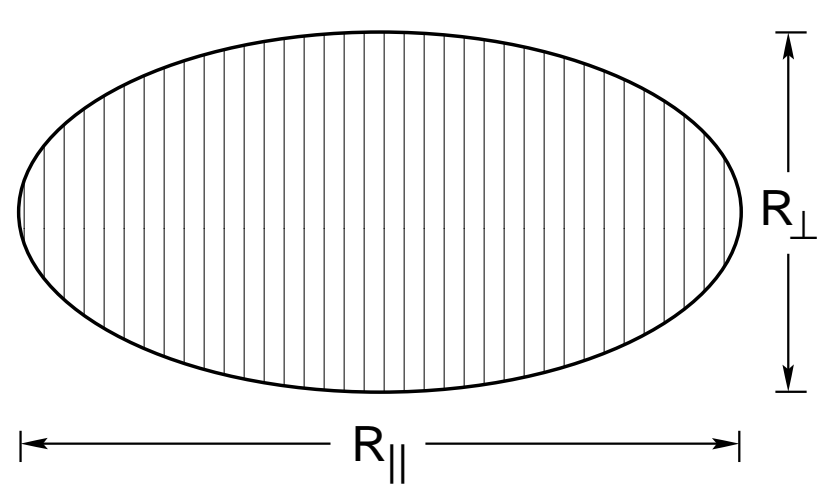

(b) 


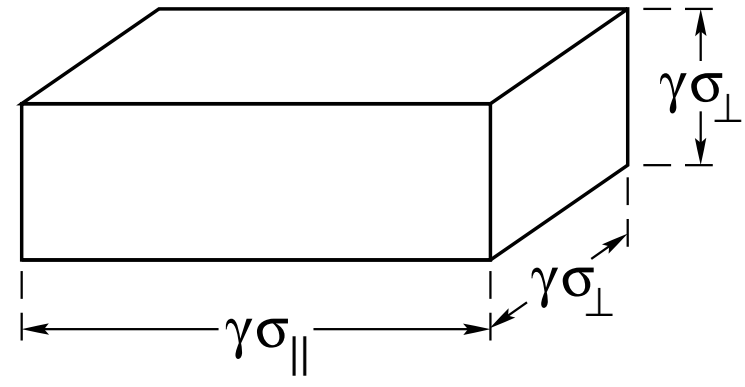

GON 4M21732.07PA 


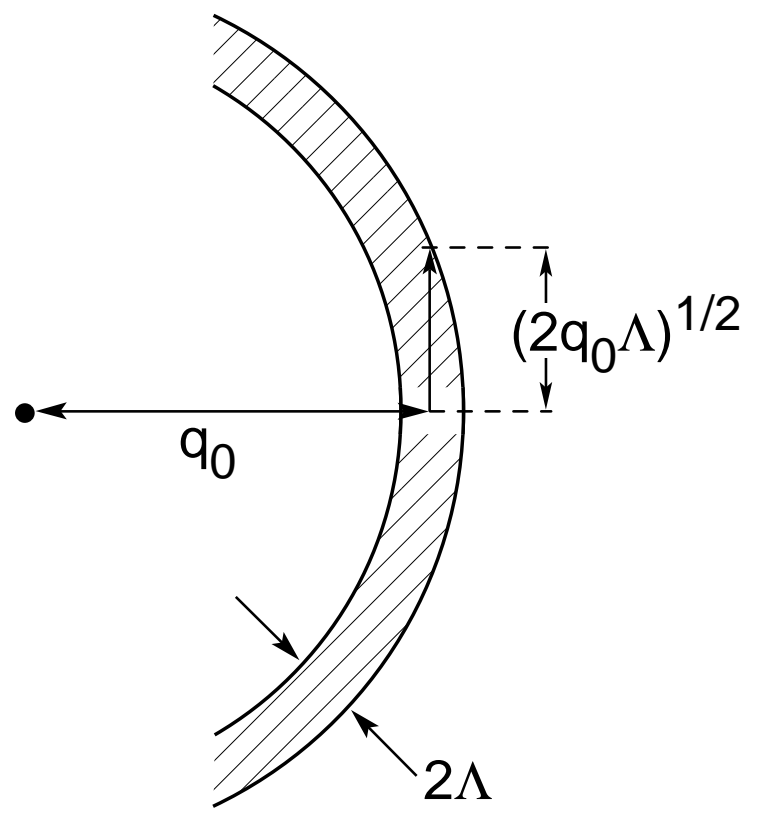

GON 4M21732 .8 BJ 


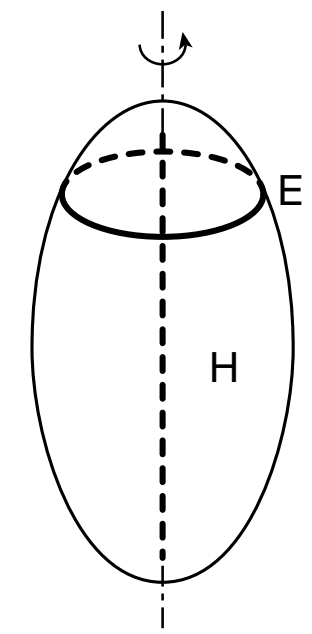

(a)

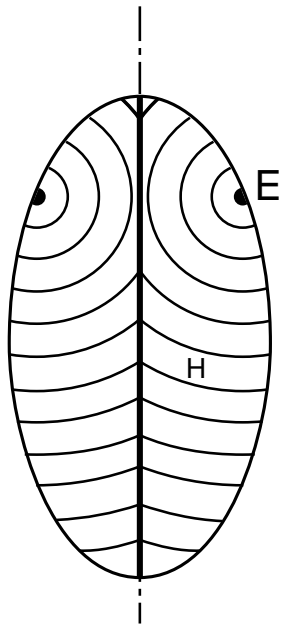

(b)

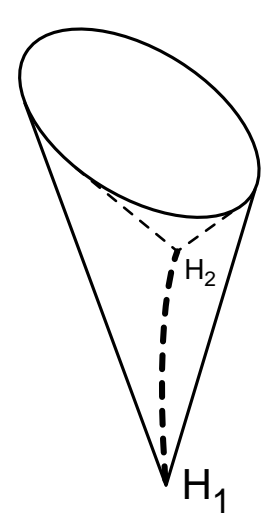

(c)

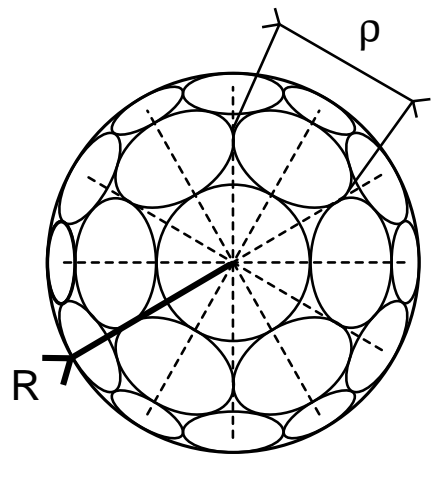

(d) 


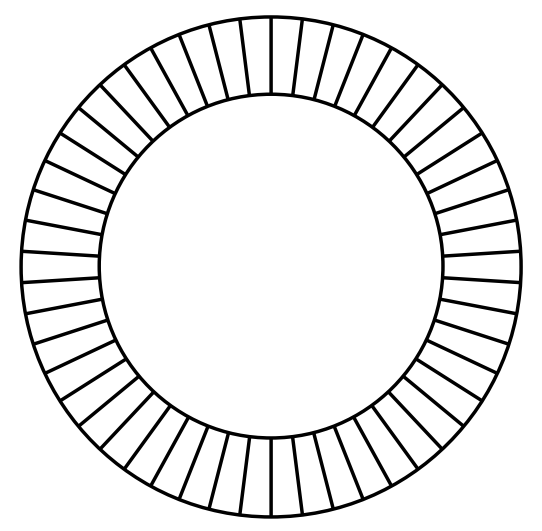

(a)

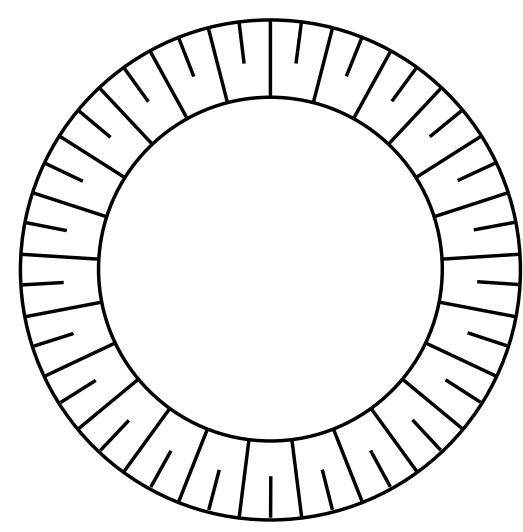

(b) 

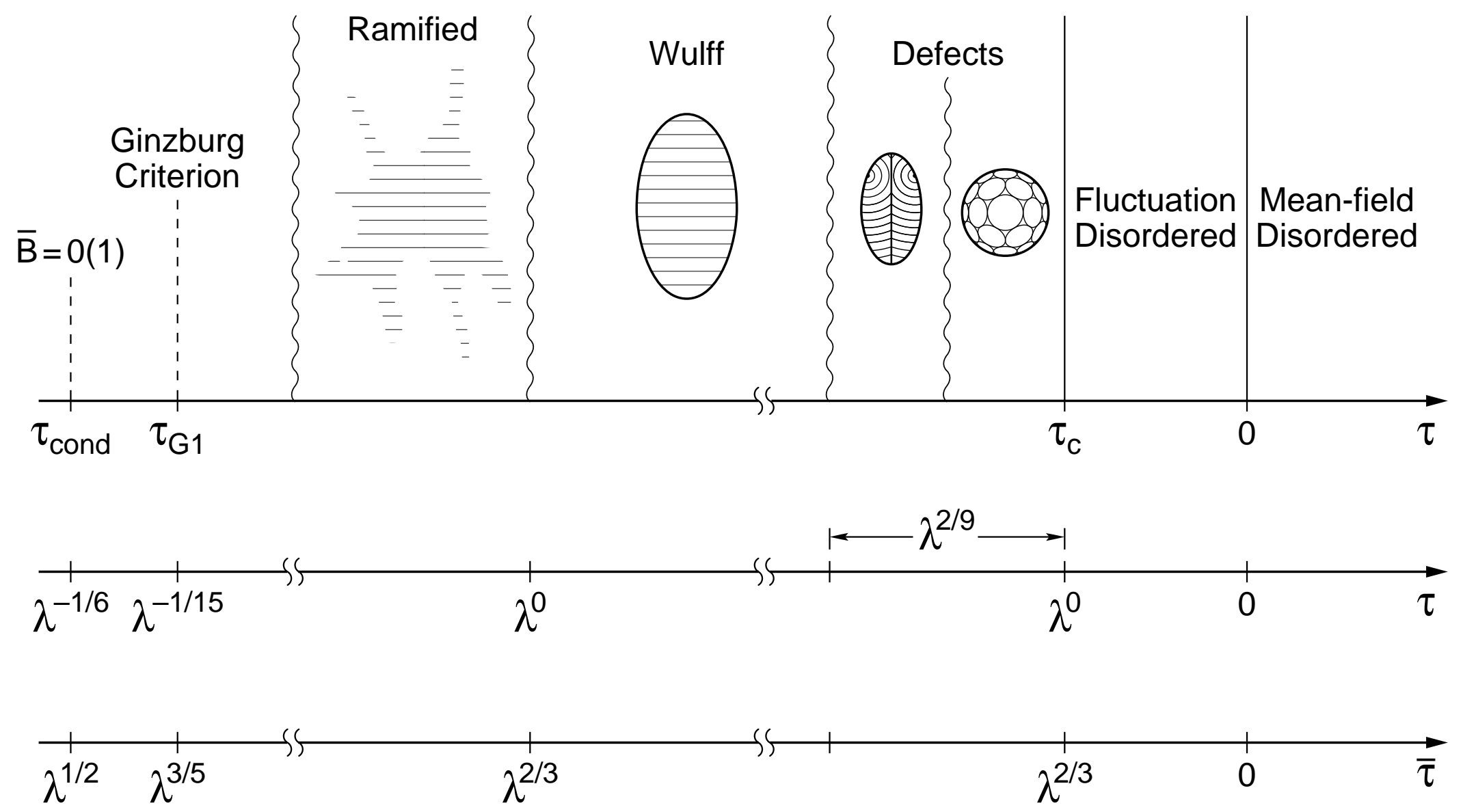


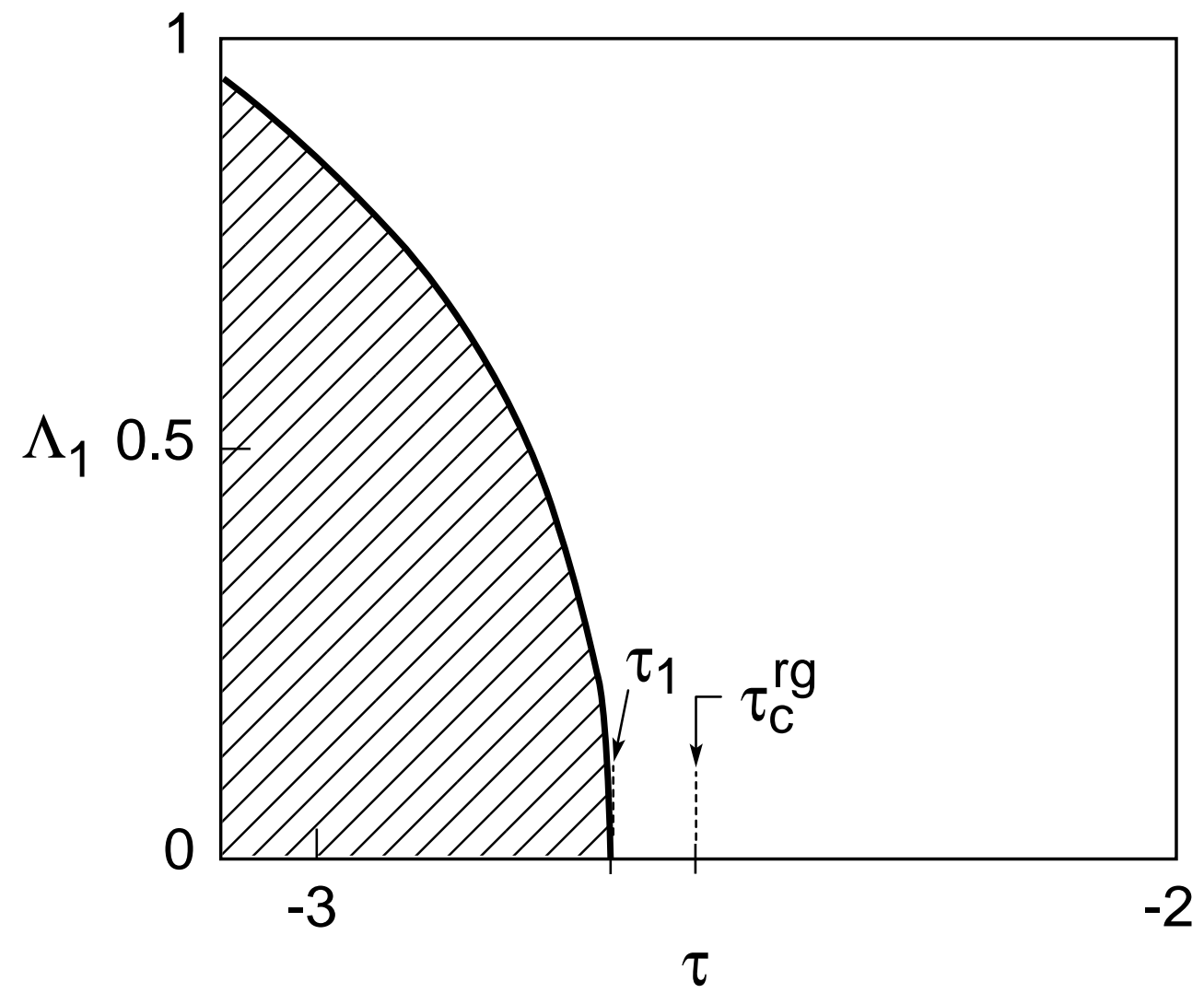




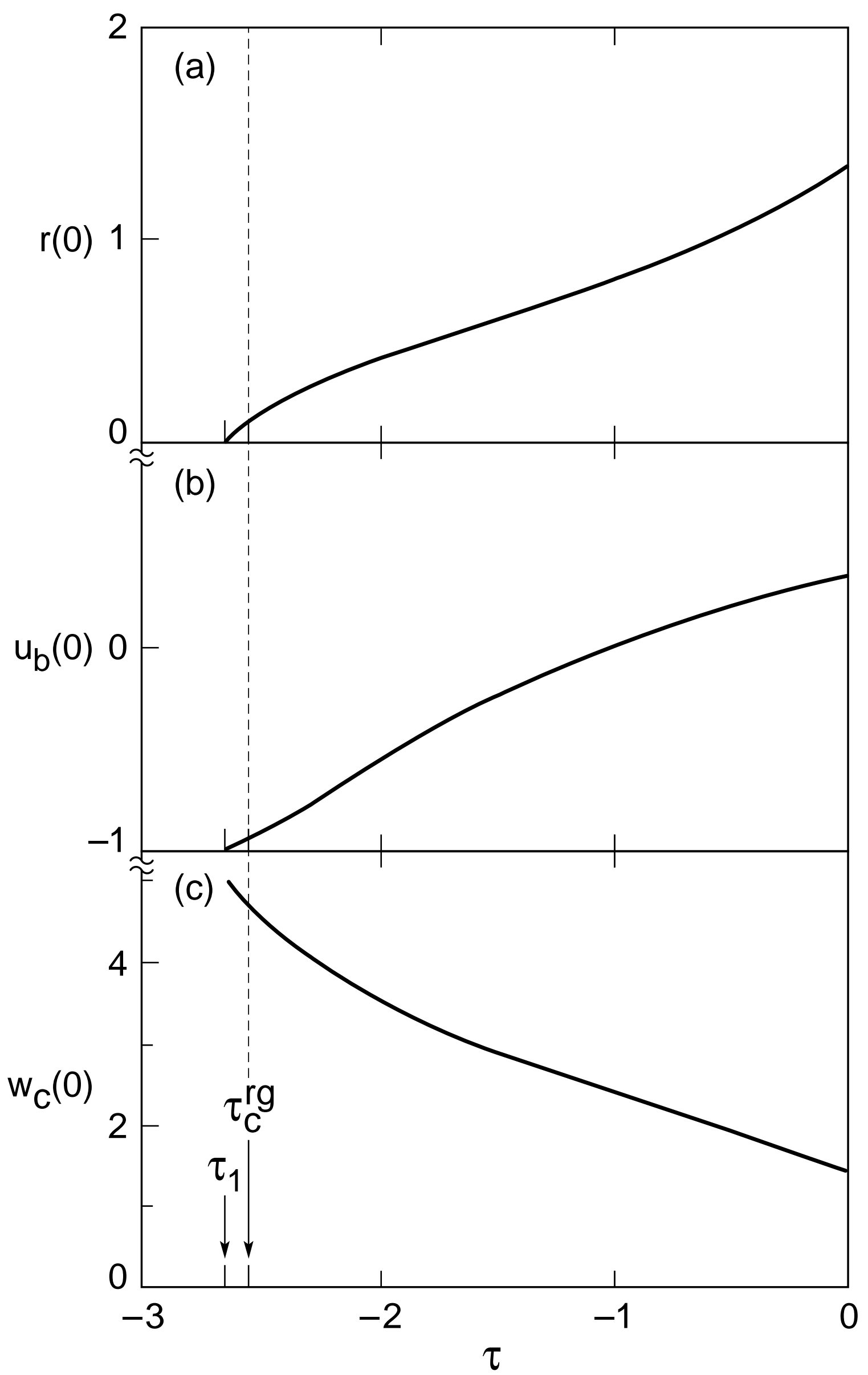

